\title{
Dwarf Galaxy Discoveries from the KMTNet Supernova Program. II. The NGC 3585 Group and Its Dynamical State*
}

\author{
Hong Soo Park ${ }^{1}$ (1), Dae-Sik Moon ${ }^{2}$ (1), Dennis Zaritsky ${ }^{3}$ (1), Sang Chul Kim ${ }^{1,4}$ (1) Youngdae Lee $^{1,5}$ (1), Sang-Mok Cha ${ }^{1,6}$, and \\ Yongseok Lee Le $^{1,6}$ \\ ${ }^{1}$ Korea Astronomy and Space Science Institute, 776, Daedeokdae-ro, Yuseong-gu, Daejeon 34055, Republic of Korea; hspark@kasi.re.kr \\ ${ }^{2}$ Dept. of Astronomy and Astrophysics, University of Toronto, 50 St. George Street, Toronto, ON M5S 3H4, Canada \\ ${ }^{3}$ Steward Observatory, University of Arizona, 933 North Cherry Avenue, Tucson, AZ 85719, USA \\ ${ }^{4}$ Korea University of Science and Technology (UST), Daejeon 34113, Republic of Korea \\ ${ }^{5}$ Department of Astronomy and Space Science, Chungnam National University, 99 Daehak-ro, Daejeon 34134, Republic of Korea \\ ${ }^{6}$ School of Space Research, Kyung Hee University, Yongin, Kyeonggi 17104, Republic of Korea \\ Received 2019 January 11; revised 2019 September 23; accepted 2019 September 23; published 2019 November 1
}

\begin{abstract}
We present our discovery and analysis of dwarf galaxies in the NGC 3585 galaxy group by the Korea Microlensing Telescope Network (KMTNet) Supernova Program. Using deep stack images reaching $\simeq 28 \operatorname{mag}^{\operatorname{arcsec}^{-2}}$ in $B V I$, we discovered 46 dwarf galaxy candidates distributed in a field of 7 square degrees. The dwarf galaxy candidates exhibit a central surface brightness as faint as $\mu_{0, V}=26.2 \mathrm{mag} \operatorname{arcsec}^{-2}$, with effective radii larger than $150 \mathrm{pc}$ and total absolute magnitudes brighter than $M_{V} \approx-10 \mathrm{mag}$, if at the distance of NGC 3585 . The dwarf galaxy surface number density decreases with projected distance from NGC 3585 . We estimate the background contamination to be about $20 \%$ based both on the number density profile and on diffuse galaxy counts in a control field. The dwarf galaxy colors and Sérsic structural parameters are consistent with those found for other dwarf galaxies. Unusually, there is no indication of a change in color or brightness in the dwarf galaxy candidates with projected distance from the group center. Approximately $20 \%$ of the candidates contain an unresolved nucleus. The nucleated fraction is larger for brighter (and redder) galaxies, but is independent of distance from the group center. We identify four ultra-diffuse galaxy candidates, all near the group center. We interpret these spatial properties as suggesting that the NGC 3585 group might be dynamically younger than the typical group. The galaxy luminosity function of the NGC 3585 group has a faint-end slope of $\alpha \approx-1.39$, which is roughly consistent with the slopes found for other nearby groups. The possible dependence of the slope on global group properties is still unclear and continues to motivate our homogeneous deep survey of dozens of nearby groups.
\end{abstract}

Key words: galaxies: dwarf - galaxies: groups: individual (NGC 3585)

\section{Introduction}

Dwarf galaxies in galaxy groups are excellent tools for exploring galaxy formation and evolution in the critical intermediate-density environments (Benson et al. 2002; Behroozi et al. 2010; Cooper et al. 2010; Presotto et al. 2012; Wetzel et al. 2012) and detailed predictions of cosmological models (Klypin et al. 1999; Moore et al. 1999; Trentham \& Tully 2002). Dwarf galaxy studies have traditionally focused on the Local Group (LG). However, technological advances have enabled wide-field surveys for low surface brightness dwarf galaxies at larger distances (McConnachie 2012; Bechtol et al. 2015; Drlica-Wagner et al. 2015). For example, access to wide-field facilities ( $\beth^{\circ} \square^{\circ}$ such as with the Canada-France-Hawaii Telescope and the Blanco $4 \mathrm{~m}$ Telescope) allows investigators to measure the luminosity function (LF) in nearby $(D \lesssim 10 \mathrm{Mpc})$ groups to $M_{V} \sim$ -10 mag (e.g., M101, Merritt et al. 2014; Bennet et al. 2017; Müller et al. 2017b; M96, Müller et al. 2018; M81, Chiboucas et al. 2009; M83, Müller et al. 2015; and NGC 5128, Crnojević et al. 2016; Müller et al. 2017a).

With these data, we can begin to compare key properties of dwarf galaxies, such as the range of structural parameters, the incidence of nucleated or ultra-diffuse galaxies (UDGs), the LF, and the spatial distribution, in the set of nearby groups (Zabludoff \& Mulchaey 2000; Chiboucas et al. 2009;

\footnotetext{
* Based on data collected at the KMTNet Telescopes.
}

Trentham \& Tully 2009; Merritt et al. 2014; Müller et al. 2015, 2017b, 2018; van Dokkum et al. 2015; Crnojević et al. 2016; Park et al. 2017) and to the properties of dwarfs found in the field and cluster environment (Young et al. 2014; Janz et al. 2016; Lee et al. 2018) to search for the effect of environmental influences. However, despite all of these recent developments, it is still difficult to obtain a consistent overview because comparisons are hindered by systematic differences in data quality, selection criteria, and technical methodology among the studies.

Several ongoing surveys are attempting to address this topic. Recently, Geha et al. (2017) introduced the survey called Satellites Around Galactic Analogs (SAGA), which aims to investigate the distribution of dwarf galaxies around 100 Milky Way (MW)-like systems at $20-40 \mathrm{Mpc}$ down to a satellite luminosity of $M_{r} \approx-12 \mathrm{mag}$. They reported the results from the study of eight MW-analog hosts, including spectroscopically confirmed complete dwarf galaxy LFs using photometry based on the gri bands of the Sloan Digital Sky Survey (SDSS). In another survey, Greco et al. (2018) presented 800 faint dwarf galaxies identified in $200 \mathrm{deg}^{2}$ of the Hyper Suprime-Cam Subaru Strategic Program (HSC-SSP), a program with a plan of eventually covering $1400 \mathrm{deg}^{2}$ with grizy bands during 300 nights (Aihara et al. 2018). Previously, Merritt et al. (2014) reported the result of observing the faint dwarf galaxies around the M101 galaxy using the observations taken with the Dragonfly Telephoto Array. Based on the results from other 
Dragonfly observations, several studies have reported on the properties of nearby groups (Danieli et al. 2017; Cohen et al. 2018). Other relevant studies are discussed in more detail farther below. Each study, however, has its own strengths and weaknesses. For example, while spectroscopic information is valuable in that it helps confirm membership, it also results in a brighter limit on the galaxies that are considered as satellites (e.g., SAGA). Other times, high sensitivity to low surface brightness objects comes at the expense of angular resolution, which can limit the study of the structural properties of the galaxies (e.g., Dragonfly). Still other times, the limitation is something as simple as limited hemispheric coverage. While the last may seem to be minor, it can be important in some contexts. In our area of interest, going as far down the LF as possible in groups, the suitable objects in the sky are limited, and accessing both hemispheres is helpful.

We are conducting a systematic study of dwarf galaxies in nearby galaxy groups using data from the Korea Microlensing Telescope Network (KMTNet) Supernova Program (KSP; Moon et al. 2016). The KSP is a program to search for supernovae and optical transients (He et al. 2016; Antoniadis et al. 2017; Brown et al. 2018; Afsariardchi et al. 2019; Lee et al. 2019) using three $1.6 \mathrm{~m}$ telescopes located at the Cerro Tololo Inter-American Observatory (CTIO, Chile), the South African Astronomical Observatory (SAAO, South Africa), and the Siding Spring Observatory (SSO, Australia). When several hundred KSP images in each field are stacked, the program provides deep $B V I$ images reaching a sensitivity of about $28 \mathrm{mag} \mathrm{arcsec}^{-2}$ within a 1 ." 5 radius aperture. The KSP dwarf galaxy survey investigates the properties of group dwarf galaxies down to a total absolute magnitude $M_{V} \sim-10$ mag using multiband ( $\left.B V I\right)$ imaging. Our survey area covers non-bulge seasons, $22^{\mathrm{h}}<$ R.A. $<24^{\mathrm{h}}$ and $0^{\mathrm{h}}<$ R.A. $<14^{\mathrm{h}}$ for decl. $<0^{\circ}$. Our target fields normally have a main galaxy that is brighter than $M_{V} \sim-20$ mag and relatively nearby, $D<20 \mathrm{Mpc}$. Additionally, we select the groups with a velocity dispersion, $\sigma_{\text {group }}$, that is $>60 \mathrm{~km} \mathrm{~s}^{-1}$ or a total mass that satisfies $\log \left(M / M_{\odot}\right)>12.0$ (Makarov \& Karachentsev 2011). We are observing more than 30 such galaxy groups up to at least 2023, when the commissioning season for the KMTNet Phase-2 is scheduled to close. Normally, we observe two fields for each targeted group, which are observed through two seasons and overlap so that the main galaxy is included in one chip (see Section 2 in detail). This results in coverage where the maximum projected radial distance from the group main galaxy is roughly $>0.5 \mathrm{Mpc}$. Our aims are to (1) identify new (and previously known) dwarf galaxies, (2) identify any cases with unusual structures (e.g., nucleated dwarf galaxies, UDGs, star clusters), and (3) uncover how group properties relate to the properties of the satellite population, such as the spatial distribution and LF. In our first paper describing results from these data (Park et al. 2017), we reported our discovery and analysis of $\sim 30$ dwarf galaxy candidates toward NGC 2784 . The eventual complete analysis of the KSP data will result in homogeneous results that can be compared for $>30$ nearby groups.

In this paper, we report on the discovery and analysis of dwarf galaxy candidates in the NGC 3585 field using stacked KSP images. The NGC 3585 group is known to include NGC 3585 itself, which is an E6 galaxy and the brightest galaxy in the group, and at least eight other galaxies with consistent radial velocities (Makarov \& Karachentsev 2011; Tully et al. 2013). We adopt a distance to this group of $20.4 \mathrm{Mpc}$ that was

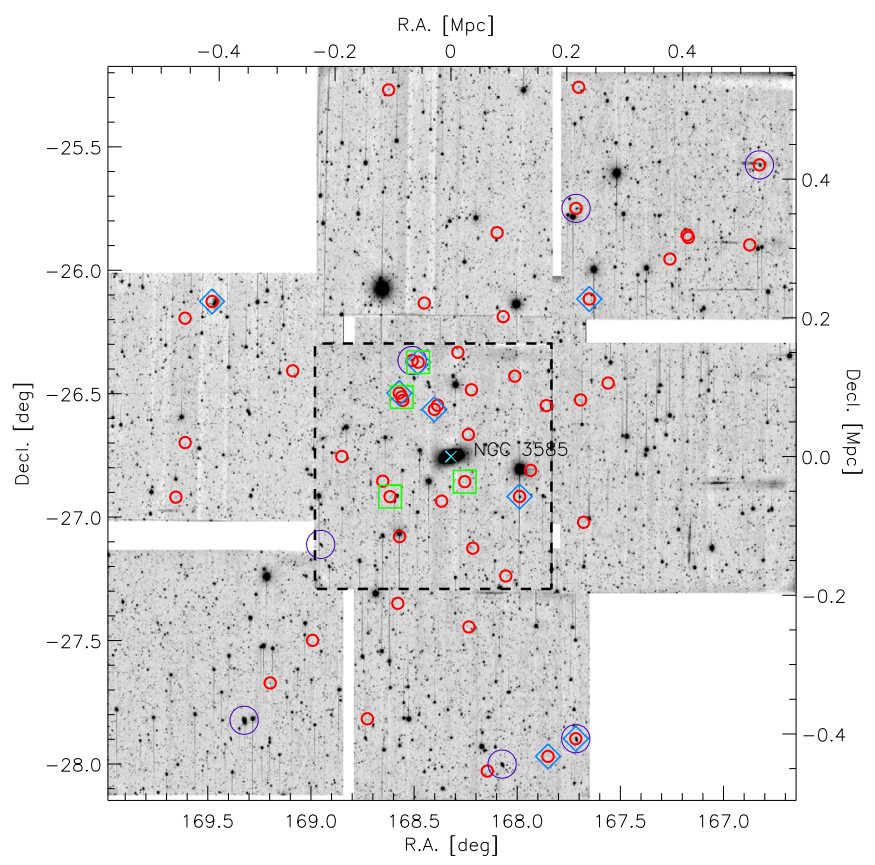

Figure 1. I-band stacked image of the entire NGC 3585 field observed by KMTNet. The red open circles are our dwarf galaxy candidates. The large dashed square represents one chip of the N3585-1 field with an area of approximately $1^{\circ} \times 1^{\circ}(0.36 \mathrm{Mpc} \times 0.36 \mathrm{Mpc})$. The large purple open circles indicate the galaxies with radial velocities similar to that of NGC 3585 . The blue diamonds and green boxes are the nucleated dwarf galaxy candidates and the UDG candidates classified in this study, respectively. The cross indicates the center of the NGC 3585 galaxy.

estimated using the surface brightness fluctuation (SBF) method (Tully et al. 2013). In Section 2 we present the observations and data reduction. In Section 3 we describe our search and the photometric results for the dwarf galaxy candidates. In Section 4 we discuss the properties of dwarf galaxy candidates detected in the NGC 3585 group and compare them to those of dwarfs in other groups.

\section{Observations and Data Reduction}

We use $B V I$ observations of two fields including NGC 3585 from the KMTNet Supernova Program using the three KMTNet telescopes (Kim \& Lee 2016) from 2016 January to 2017 June. The wide-field CCD camera installed in each telescope covers $4 \square^{\circ}\left(2^{\circ} \times 2^{\circ}\right)$ with 0 "! 4 pixel $^{-1}$ scale. The detector is a mosaic of four chips, and each chip has a $9 \mathrm{~K} \times 9 \mathrm{~K}$ array with with a small gap between the chips. Each chip corresponds to a $1^{\circ} \times 1^{\circ}$ field of view. $B V I$ filters were used, and the exposure time in each image was fixed to be $60 \mathrm{~s}$. In Figure 1 we show the $I$-band stacked image for the two fields observed toward NGC 3585, which overlap in an area of approximately $1^{\circ} \times 1^{\circ}(0.36 \mathrm{Mpc} \times 0.36 \mathrm{Mpc}$ at the distance of the NGC 3585), so the net size of the field of view is 7 square degrees. In addition, we also show in Figure 1 the locations of our newly discovered dwarf galaxy candidates. One region, N3585-1 (included as the dashed square in Figure 1), was observed approximately 600 times in each filter, while the other region, N3585-2 (four images in the lower left corner), was observed about half as many times. As a result, the former images are approximately 0.3 mag $\operatorname{arcsec}^{-2}$ deeper than the latter in I-band surface brightness.

Following the standard preprocessing procedure, which includes a crosstalk correction, overscan subtraction, and 
Table 1

Observing Log for Stack Images

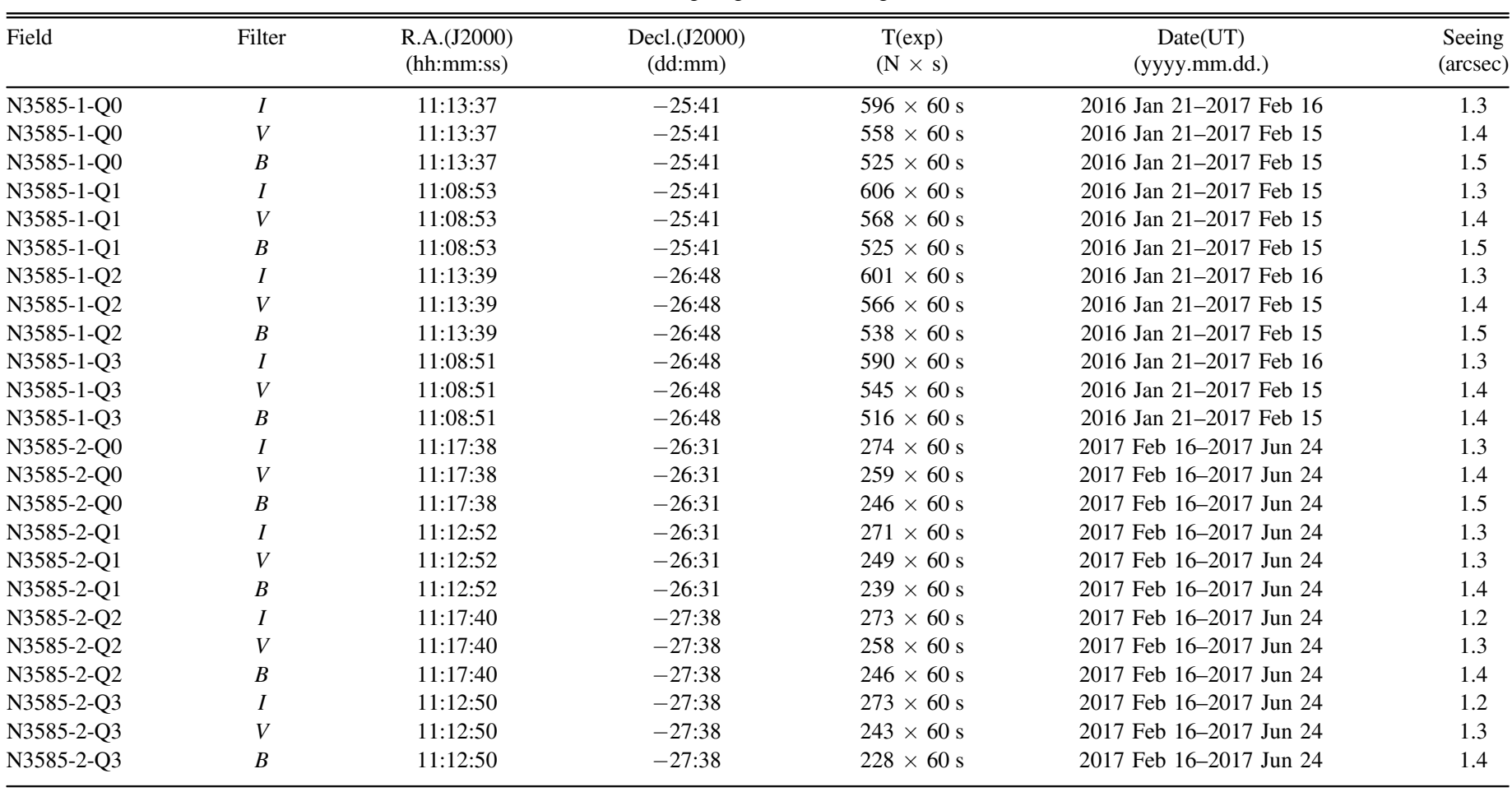

Table 2

Standardization of Instrumental Magnitudes

\begin{tabular}{lcccc}
\hline \hline Field & $c(B-V)$ & $\begin{array}{c}\text { Zero(B) } \\
(\mathrm{mag})\end{array}$ & $\begin{array}{c}\text { Rms }(B) \\
(\mathrm{mag})\end{array}$ & $\begin{array}{c}\text { Zero(V) } \\
(\mathrm{mag})\end{array}$ \\
\hline N3585-1-Q0 & $0.32 \pm 0.02$ & $28.09 \pm 0.01$ & 0.04 & $28.11 \pm 0.03$ \\
N3585-1-Q1 & $0.28 \pm 0.01$ & $28.15 \pm 0.01$ & 0.02 & $28.12 \pm 0.03$ \\
N3585-1-Q2 & $0.28 \pm 0.01$ & $28.17 \pm 0.01$ & 0.02 & $28.14 \pm 0.03$ \\
N3585-1-Q3 & $0.26 \pm 0.02$ & $28.14 \pm 0.01$ & 0.03 & $28.11 \pm 0.04$ \\
N3585-2-Q0 & $0.25 \pm 0.02$ & $28.26 \pm 0.02$ & 0.03 & $28.19 \pm 0.04$ \\
N3585-2-Q1 & $0.27 \pm 0.01$ & $28.26 \pm 0.01$ & 0.02 & $28.20 \pm 0.03$ \\
N3585-2-Q2 & $0.27 \pm 0.01$ & $28.22 \pm 0.01$ & 0.02 & $28.18 \pm 0.03$ \\
N3585-2-Q3 & $0.27 \pm 0.01$ & $28.20 \pm 0.01$ & 0.03 & $28.03 \pm 0.03$ \\
\end{tabular}

flat-fielding, we obtain an astrometric solution using the $S C A M P$ program (Bertin 2006). We then stack the images that have better than $2^{\prime \prime}$ seeing using the SWARP program (Bertin et al. 2002) to produce the images that we analyze and present here (see Section 2 of Park et al. 2017 for more details). The seeing in the resultant stacked images is better than 1.15 in all bands. Details of the observations are listed in Table 1.

We obtain photometric solutions for our stack images using standard stars in the AAVSO Photometric All-Sky Survey (APASS) catalog (APASS 2015). As in Park et al. (2017; see their Section 2 and Figure 2 for details), we make a color correction in transforming instrumental $B$-band magnitudes to standard values: $B=b+c(B-V)+$ zero. Here, we use 0.27 for the approximate color coefficient $(c)$, which we obtained from about 150 stars per chip (the value of $c$ is almost identical to what we obtained for the NGC 2784 fields; see Park et al. 2017). On the other hand, we apply only zero-point offsets for the transformation of the $V$ and $I$ bands. The zero-points for each band are $28.2 \pm 0.03,28.2 \pm 0.03$, and $28.1 \pm 0.05$ mag for the $B, V$, and $I$ bands, respectively. We list the values we use for the standardization of the instrumental magnitudes in each chip in Table 2. We note that for the $I$-band calibration, we convert the Sloan $r$ and $i$ magnitudes into the APASS catalog to $I$-band magnitudes using the equation $I=r-$ $1.2444(r-i)-0.3820$ from Lupton (2005). We then correct our galaxy photometry for Galactic extinction in this field using $A_{B}=0.231, \quad A_{V}=0.175$, and $A_{I}=0.096$ (Schlafly \& Finkbeiner 2011). ${ }^{7}$

\section{New Dwarf Galaxy Candidates and Basic Properties}

\subsection{Search for Dwarf Galaxy Candidates}

To find dwarf galaxy candidates in the two NGC 3585 fields, we visually select dwarf galaxy candidates by identifying

\footnotetext{
7 To clarify some confusing notation, we only apply extinction corrections to total magnitudes and colors. When these corrections were applied, we signify this with the subscript 0 , as in $(B-V)_{0}$. When no subscript is included, the corrections were not applied. Second, for historical consistency, we also use the subscript 0 to refer to central values, as in the central surface brightness, $\mu_{0}$. The subscript here does not imply that an extinction correction was applied. Third, we also use the subscript 0 on the scaling radius, $r_{0}$, which is different from the effective radius, $r_{e}$. The relationship between the scaling radius and $r_{e}$ depends on the Sérsic index, $n$.
} 
diffuse sources (see Park et al. 2017 for details). For dwarf galaxy candidates in the NGC 3585 group, the $\sim 20 \mathrm{Mpc}$ distance, or $(m-M) \sim 31.5$ mag, means that the stellar populations will present as unresolved diffuse features because even the stars on the tip of the red giant branch (TRGB), one of the brightest stellar populations, cannot be resolved. In our stacked image, which has a point-source magnitude limit of about $24 \mathrm{mag}$, the TRGB stars with $M_{I}=-4.0 \mathrm{mag}$ (Lee et al. 1993) have an apparent magnitude of about 27.5 mag. We use primarily the $I$-band image to identify galaxies because it is the deepest of the three bands for a given exposure time and because most dwarf galaxies have a red color, $(V-I)>0.5$ (see Section 3.4). We use the $B$ - and $V$-band images, as well as RGB color images made from the three bands, to rule out artifacts.

To enhance faint surface brightness features, we set the dynamical scale range on a display screen (e.g., DS9) such that the lowest and highest intensities are approximately $-3 \sigma$ and $10 \sigma$ from the mean, where $\sigma$ refers to the scatter of the sky background. We select any diffuse sources that are approximately larger than $10^{\prime \prime}$ as dwarf galaxy candidates, and currently reject sources with special features (e.g., spiral or bulge) except for those with a point source at the center, which are classified as nucleated dwarf galaxy candidates (see Section 4.2). To minimize biases in the detection and incompleteness, three authors (H.S.P., S.C.K., and Y.L.) searched the images independently and then compared the results. For candidates selected by only one or two classifiers, all three classifiers reviewed the object together and decided whether to retain or reject the candidate basically based on the above criteria. The retained candidates are roughly $20 \%$ of all the candidates that were finally accepted.

For the regions near NGC 3585 itself, we use an image where we have subtracted a model for the galaxy constructed with the IRAF/BMODEL task. The model is constructed using the output from the IRAF/ELLIPSE task, using nonlinear steps along the semimajor axis and floating ellipticity and position angle, with the brightness profile measured using median isophotal fluxes after interactively masking bright objects (e.g., foreground stars or background galaxies) and applying two iterations of $3 \sigma$ clipping. This model subtraction was only performed on the $0^{\circ} .25 \times 0^{\circ} .25$ area centered on NGC 3585 galaxy in the N3585-1 field. From our visual inspection of the subtracted image we conclude that the model fitting is adequate to within $1^{\prime}$ from the center of NGC 3585.

Despite the difference in depth between the two observed fields, we recover the same candidates in both fields within the overlapping region. We conclude that our ability to recover dwarfs is not set by the point-source limiting magnitude of each field, but rather by other factors such as scattered light from bright stars, image defects, and a spatially variable background. Because none of these limiting factors are affected by the modest difference in exposure time between the two fields, we treat the two fields in the same way. We also searched for dwarf galaxy candidates in the image stacked within the overlapping region, but did not find any new diffuse sources. In total, we identify 46 such diffuse objects and plot their positions in Figure 1.

We then use the NASA Extragalactic Database (NED) to search for known galaxies in the NGC 3585 KMTNet fields. We find eight galaxies with existing radial velocity measurements that are compatible with membership in the NGC 3585 group as defined by being within $\pm 3 \sigma_{v}, 1220<c z<$ $1650 \mathrm{~km} \mathrm{~s}^{-1}$. We define this velocity range using the measured group velocity dispersion, $\sigma_{v}=70 \mathrm{~km} \mathrm{~s}^{-1}$ (Makarov \& Karachentsev 2011) and the radial velocity, $v=1434 \mathrm{~km} \mathrm{~s}^{-1}$ (NED). The four of these eight galaxies that have an absolute magnitude fainter than -18 mag and approximately exponential brightness profiles are dwarf galaxy group members. These galaxies are, in retrospect, detected in our KMTNet images, but do not belong to the 46 dwarf candidates. They were not included in our candidate list because they have high surface brightnesses and are much more difficult to distinguish from background galaxies. That is, these bright dwarf galaxies have an extended central region (approximately $\mu \lesssim 23$ mag $\operatorname{arcsec}^{-2}$ ) that is saturated in our displayed range, which we generally consider to be a signature of background galaxies. As such, there could be additional high surface brightness group members that are among neither the NED galaxies nor our cataloged candidates.

We also examined whether archival images, such as those from the Dark Energy Camera Legacy Survey (DECaLS) and the Hubble Space Telescope (HST), could be used to confirm our candidates and to search for new diffuse sources. Unfortunately, the survey region of the former does not overlap with our NGC 3585 field. The latter, HST, has two overlapping fields (a central region and a region $\sim 30^{\prime}$ away from the NGC 3585 galaxy). However, none of the dwarf galaxy candidates we identified lie within these fields, and we did not find any additional diffuse candidates.

We now add the four NED dwarfs to our dwarf galaxy candidate list for the remainder of this study. We use the brighter four confirmed group galaxies, which are not dwarfs, only when discussing the galaxy LF in Section 4.4. In Figure 2 we present the $I$-band grayscale images of all the dwarf galaxy candidates and the additional four confirmed dwarfs. They tend to be diffuse with a variety of sizes and morphologies. Some show concentrated nucleated emission.

We estimate the limiting brightness of our I-band images using a completeness test where we create and attempt to recover several hundred artificial galaxies. We create the galaxies and add them to our I-band images of the deep field (N3585-1-Q2) and the shallow field (N3585-2-Q1), including the NGC 3585 galaxy, using the mkobjects task in the IRAF/ artdata package. In our modeling, we adopt surface brightness profiles ranging from $n=0.6$ to $n=1$ and an effective radius distribution that matches the effective radius-magnitude relation of the NGC 3585 dwarf galaxy candidates in Figure 6 (see Section 3.4). Based on reproducing our the visual inspection procedure, we determine that we are $90 \%$ complete down to $I \approx 19.8 \mathrm{mag}$ in the deep field $\left(M_{V} \approx-11.0\right.$ mag at the distance of NGC 3585) and to $I \approx 19.6$ mag in the shallow field.

For a galaxy survey that targets galaxies larger than a given radius $\left(r_{\text {lim }}\right)$ with a given surface brightness $\left(\mu_{\text {lim }}\right)$, we can predict detection limits for radius or surface brightness versus total magnitude (Ferguson \& Sandage 1988; Müller et al. 2015). For our dwarf galaxy survey, in which we detect galaxies larger than $10^{\prime \prime}\left(r_{\mathrm{lim}}=5^{\prime \prime}\right)$ down to a $\mu_{\text {lim }}=28 \mathrm{mag}$ $\operatorname{arcsec}^{-2}$, the completeness boundaries are drawn as dotted curves in Figure 6, assuming that the dwarfs have exponential surface brightness profiles. Most of our dwarf galaxy candidates are located on the left side of the boundaries, as 

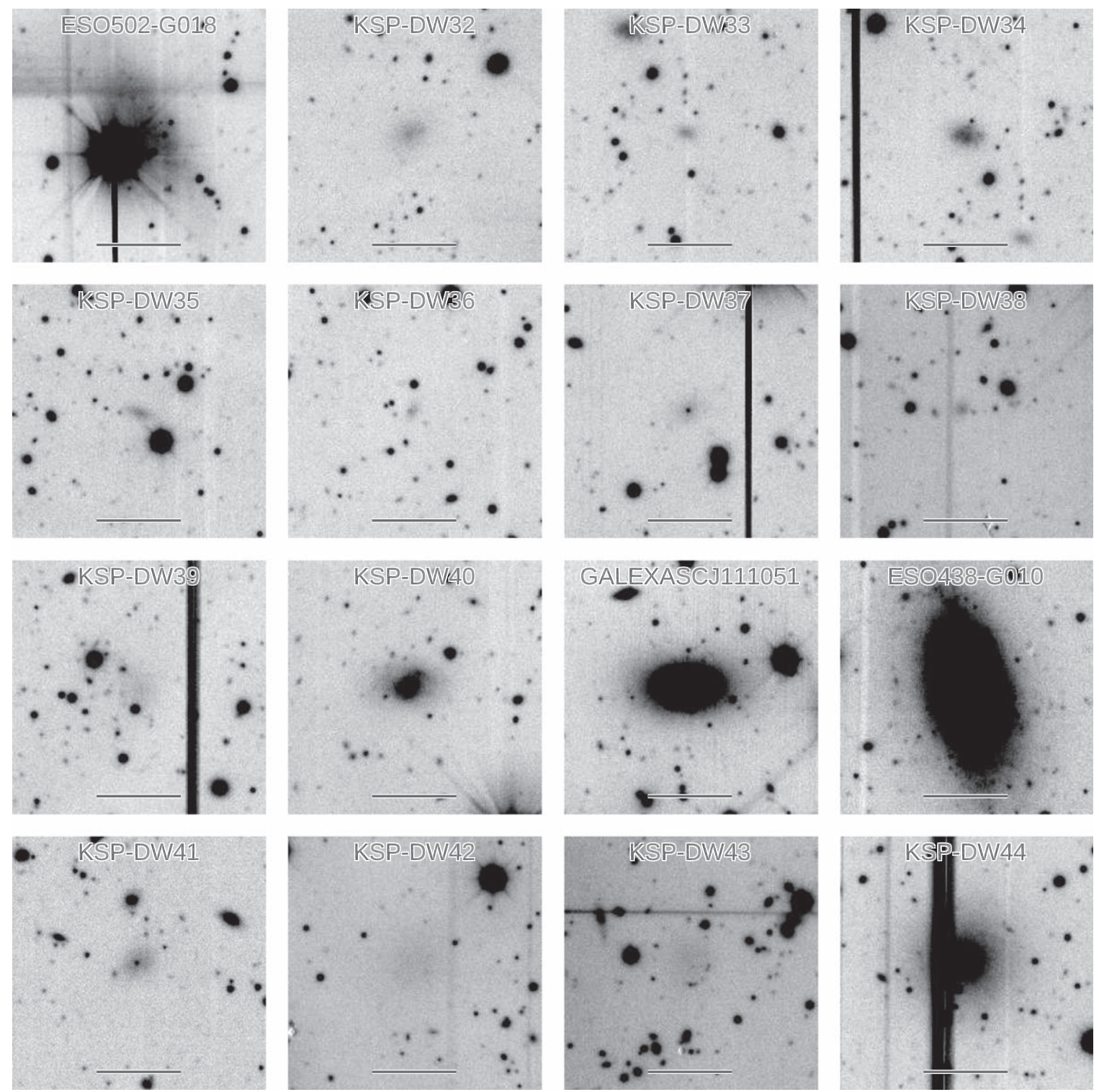

Figure 2. I-band images of the dwarf galaxy candidates in N3585 fields. The scale bar in each panel represents $30^{\prime \prime}$, and the field of view of each image is $\sim 1 ! 5 \times 1 ! 5$. North is up and east is to the left.

expected. The results from this calculation are also consistent with those from the injection of artificial galaxies.

The central region of a group is often contaminated by diffuse intragroup light (Watkins et al. 2014, 2015; Mihos et al. 2017). We now check whether diffuse intragroup light affects our detection of dwarf galaxy candidates. We compare the recovery for the inner (within $\sim 0.15 \mathrm{Mpc}$, the area that would suffer most from intragroup light) and the outer regions. The completeness difference is smaller than $5 \%$, which is within our $1 \sigma$ uncertainty. We therefore conclude that intragroup light does not affect this study.

Finally, our dwarf galaxy candidates might be confused with Galactic cirrus, which is often observed in deep optical images (Miville-Deschênes et al. 2016). Because Galactic cirrus is attributed to dust grains, we examine the WISE $12 \mu \mathrm{m}$ map of this region to help us distinguish between dwarf galaxy candidates and Galactic cirrus. Our NGC 3585 fields are located outside clear regions of Galactic cirrus that can be seen in the WISE $12 \mu \mathrm{m}$ map. In terms of color and surface brightness, Galactic cirrus has red colors $(1.3$ mag < $(g-r)_{0}<2.0 \mathrm{mag}$ and $1.5 \mathrm{mag}<(B-V)_{0}<2.2 \mathrm{mag}$; Ludwig et al. 2012), while our dwarf galaxy candidates have relatively blue colors, $0.1 \mathrm{mag}<(B-V)_{0}<1.0$ mag (see Section 3.4). Last, all of our dwarf galaxy candidates have a brighter central surface brightnesses than the surface brightness of Galactic cirrus $\left(\mu_{B}>27 \mathrm{mag} \operatorname{arcsec}^{-2}\right.$; Cortese et al. 2010). We conclude that most if not all of our dwarf galaxy candidates are not misclassified Galactic cirrus.

\subsection{Surface Photometry and Catalog}

We measure the surface brightness profiles of the 46 dwarf galaxy candidates and the 4 previously known dwarf galaxies in $B, V$, and $I$ using the IRAF/ELLIPSE task. Because many of our candidates have low surface brightness, we performed the photometry in two steps. First, we measure the brightness of the candidates without fixing the parameters (e.g., center, position angle, and ellipticity) using the ELLIPSE task on the I-band image, which is relatively deeper than other band images. We then fix the parameters to be the values measured 

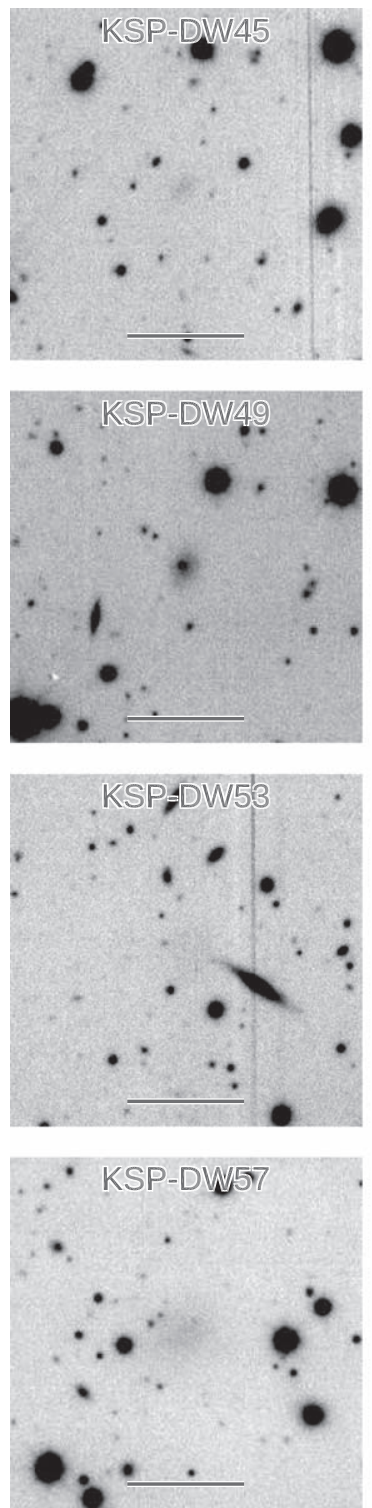
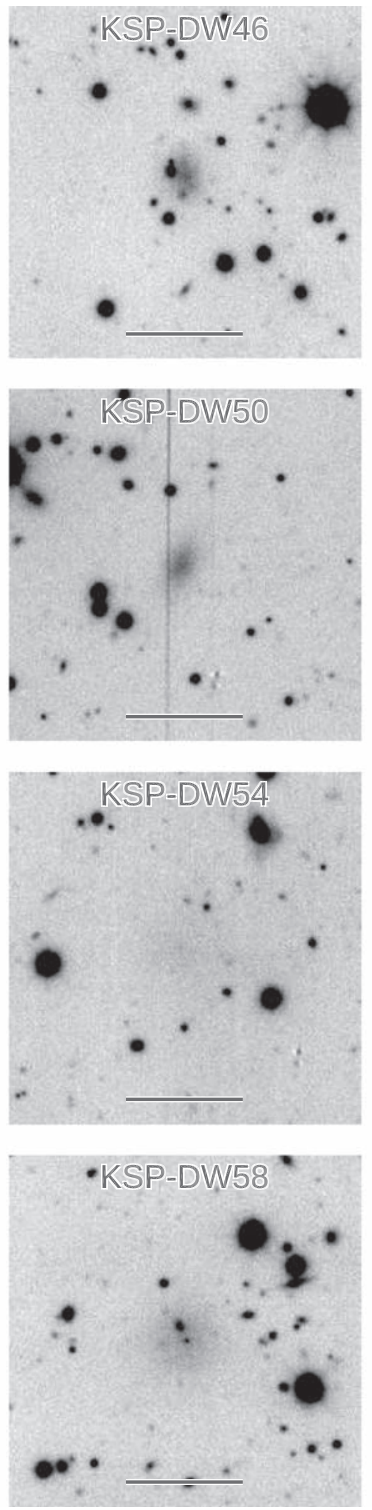
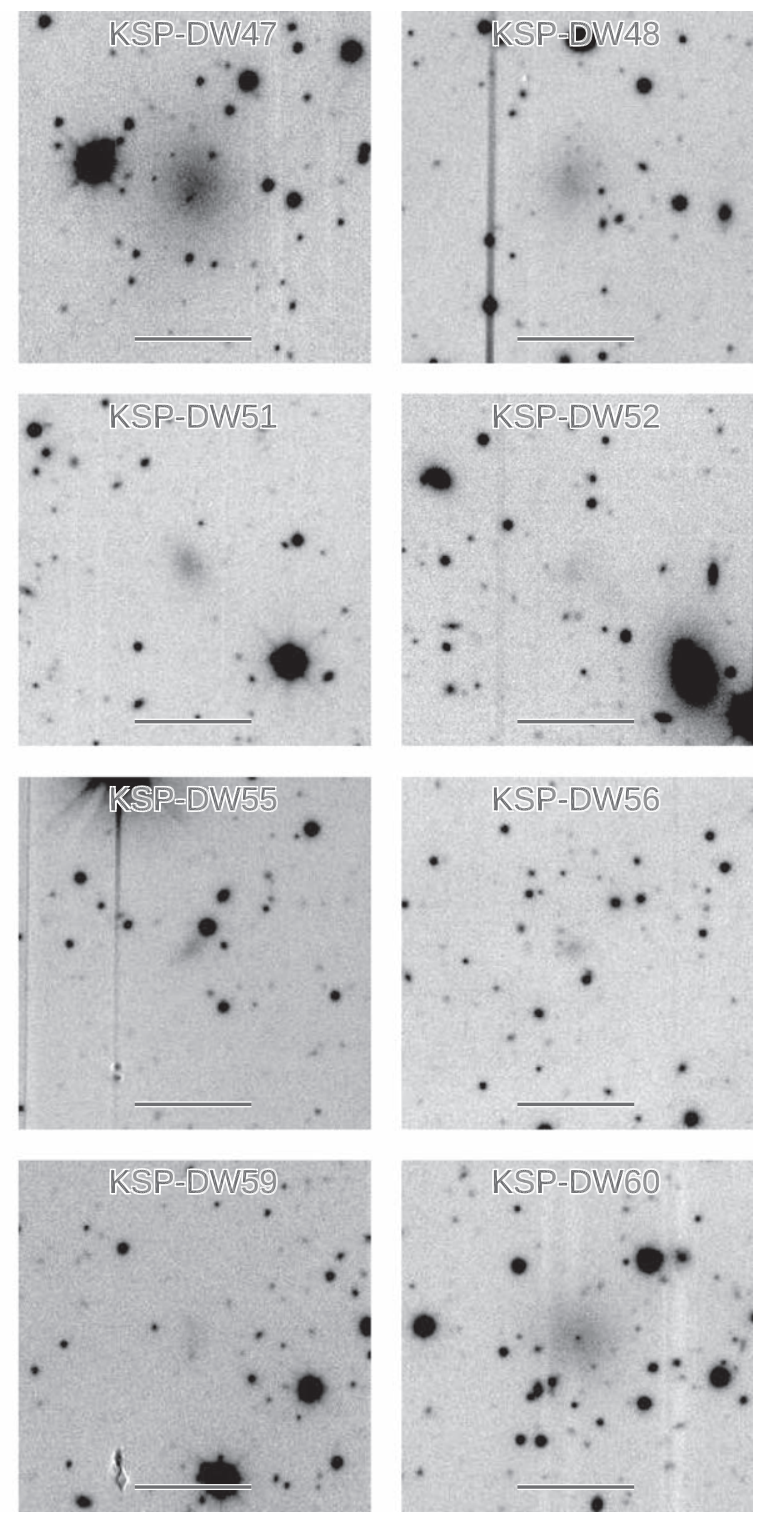

Figure 2. (Continued.)

around the resulting effective radius and measure the surface brightness profile of each candidate on the $B V I$-band images. We use 1"! 2 linear steps along the semimajor axis and measure the mean isophotal flux. The uncertainty in each measurement is determined from the combination of three sources as follows: (1) the mean error of the isophotal flux as given by ELLIPSE, (2) the variation of sky background level, which is estimated from the fluxes in several of the outermost radial bins, and (3) the variation among fitting parameters, which we estimate by calculating several hundred trials while varying the parameters according to the uncertainties of the fixed parameters returned originally by ELLISPE. Finally, we transform the instrumental surface brightness using the zero-points and color terms measured in Section 2.

In Figure 3 we show the surface brightness (upper panels) and color (lower panels) profiles of the NGC 3585 dwarf galaxy candidates. Most candidates have roughly an exponential $(n \sim 1)$ surface brightness profile and a constant color profile. The faintest surface brightnesses reach approximately $28 \mathrm{mag} \operatorname{arcsec}^{-2}$. The weighted mean colors $\left(\left\langle\mu_{(V-I)}\right\rangle\right.$ and
$\left.\left\langle\mu_{(B-V)}\right\rangle\right)$ are denoted by the blue dot-dashed and green dotted lines, respectively, in the color profile panels. We adopt the mean color as the color, $(V-I)$ and $(B-V)$, of each dwarf galaxy candidate. The color ranges are $0.3<(B-V)<1.1$ and $0.6<(V-I)<1.3$.

We use the Sérsic function $\left(\mu_{0}+1.0857\left(r / r_{0}\right)^{1 / n}\right)$, where $\mu_{0}$, $r_{0}$, and $n$ are the central surface brightness, scale length, and Sérsic curvature index, respectively, to fit the surface brightness profiles. The best fits of the Sérsic functions are presented as solid curves in Figure 3. We adopt the $1 \sigma$ statistical errors estimated from the nonlinear least-squares fitting procedure (Markwardt 2009) for the uncertainties of the Sérsic parameters. Typical uncertainties for our candidates $\left(\left\langle\sigma\left(\mu_{0, I}\right)\right\rangle \approx 0.12 \mathrm{mag}\right.$ $\operatorname{arcsec}^{-2},\left\langle\sigma\left(r_{0, I}\right)\right\rangle \approx 0$.' 59, and $\left.\left\langle\sigma\left(n_{I}\right)\right\rangle \approx 0.13\right)$ are plotted in Figure 6. All of the dwarf galaxy candidates have central surface brightness values brighter than $\mu_{0, I} \sim 25.4, \mu_{0, V} \sim 26.2$, and $\mu_{0, B} \sim 27.1 \mathrm{mag} \operatorname{arcsec}^{-2}$. They have Sérsic scale lengths of $2^{\prime \prime}<r_{0, I}<24^{\prime \prime}$ and curvature indices of $0.4<n_{I}<2.0$ with a median value of $\sim 0.8$. The best fits for dwarf galaxy candidates 

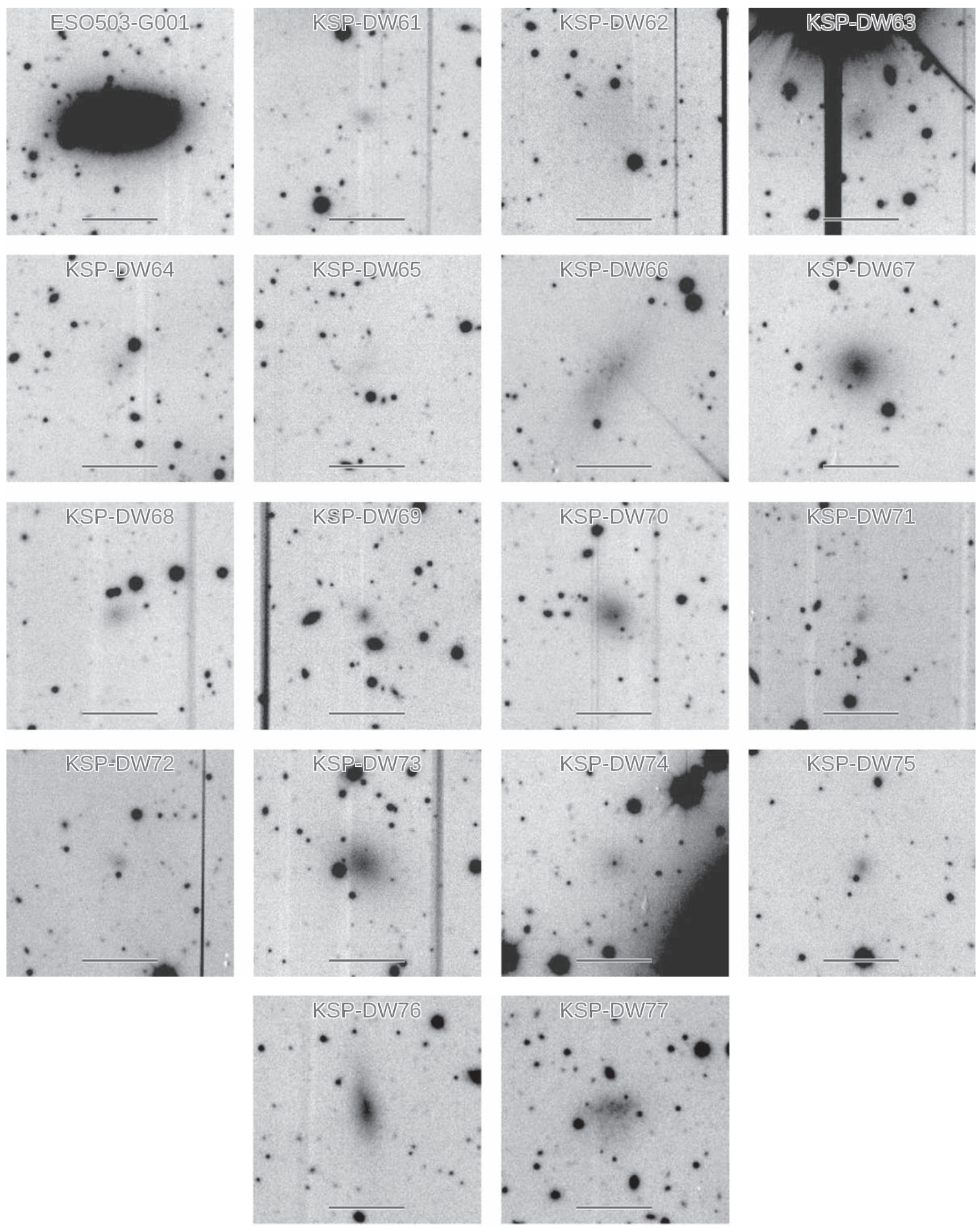

Figure 2. (Continued.)

in the overlap regions of N3585-1 and N3585-2 fields (Figure 1) are comparable, showing consistency in our measurements.

We estimate their total magnitudes and effective radii from the extrapolation of the Sérsic fits (see Section 3.1 of Chiboucas et al. 2009 for the details of the method). The faintest total magnitudes in the $B, V$, and $I$ bands are 22.4, 21.6, and $20.5 \mathrm{mag}$, respectively. The smallest effective radius $\left(r_{e, I}\right)$ in the $I$ band is $\sim 11^{\prime \prime} 5$ (corresponding to $\sim 150 \mathrm{pc}$ at the distance of NGC 3585). The uncertainties of their total magnitudes and effective radii are derived by measuring several hundred trials while varying according to the uncertainties of the Serrsic parameters. The typical values, $\langle\sigma(I)\rangle \approx 0.14 \mathrm{mag}$ and $\left\langle\sigma\left(r_{e, I}\right)\right\rangle \approx 0$." 76 , for our dwarf galaxy candidates are also plotted in Figure 6.

In Table 3 we present the photometric results for the 46 dwarf galaxy candidates in the study and for the 4 confirmed dwarfs. The first column contains the name. The second and third columns list the central coordinates. The fourth column presents the total $I$-band magnitude based on the Sersic fit. The fifth and sixth columns are the observed $(V-I)$ and $(B-V)$ 

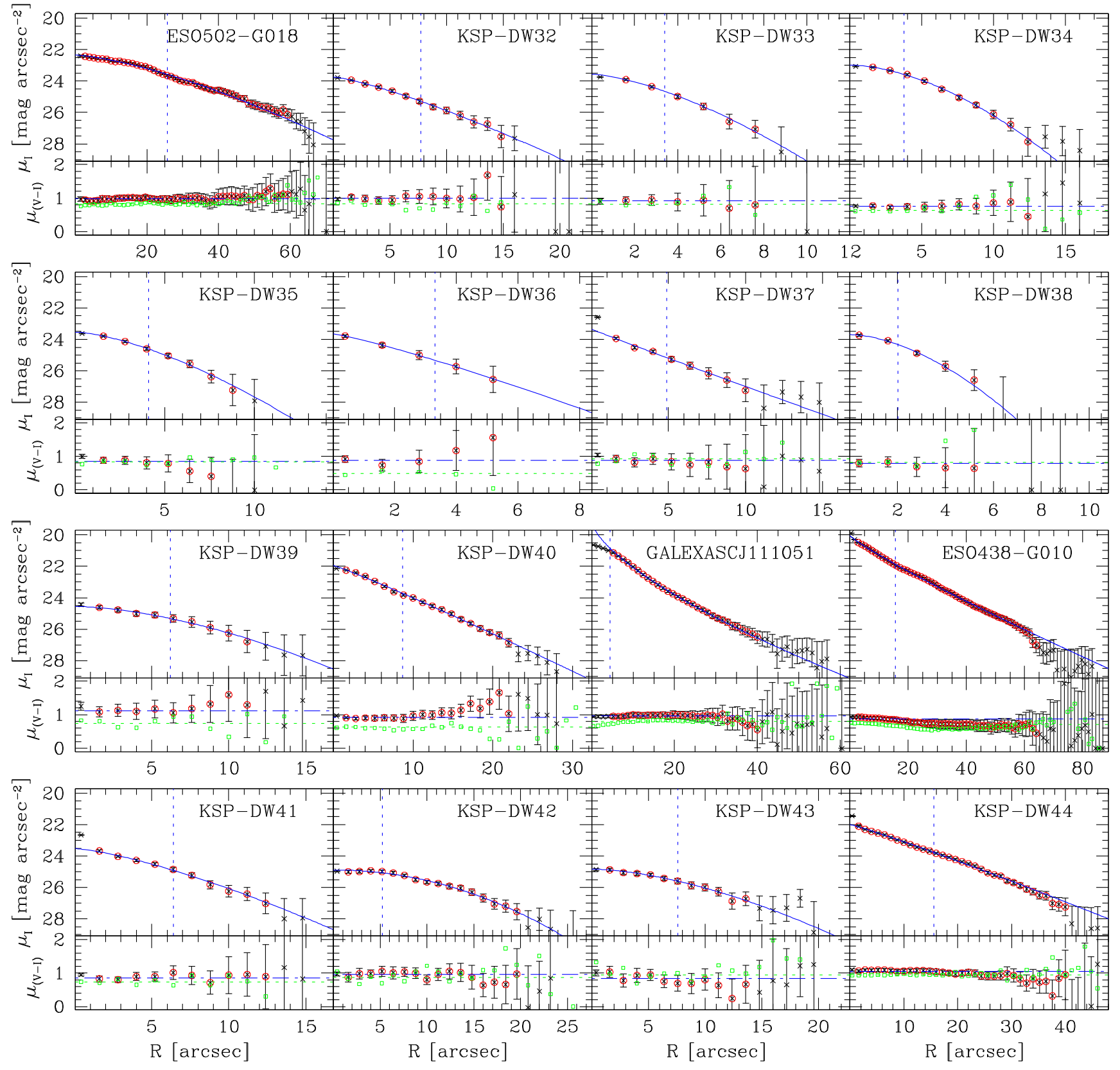

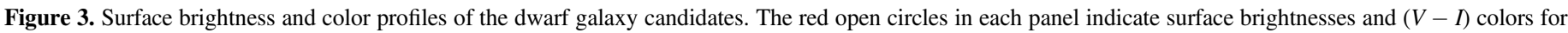

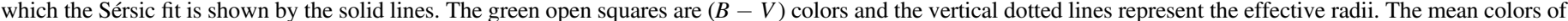
$(V-I)$ and $(B-V)$ are presented by blue dot-dashed and green dotted lines, respectively.

colors, respectively. The seventh, eigth, ninth, and tenth columns list the central surface brightness $\left(\mu_{0, I}\right)$, scale length $\left(r_{0, I}\right)$, curvature index $\left(n_{I}\right)$, and $I$-band effective radius $\left(r_{e, I}\right)$ derived from the Sérsic fit, respectively. The "N" and "U" flags in the last column indicate whether the galaxy candidate is nucleated ("N") and/or if it can be qualified as an ultra-diffuse galaxy ("U"). See Sections 4.2 and 4.3 for the details of these classifications.

For the purpose of constructing the luminosity function (Section 4.4), we also photometered the four bright group galaxies (see Section 3.1 for details). The resulting photometry of the bright galaxies is as follows: NGC $3585\left(M_{V}=-22.52\right)$, UGCA230 $\left(M_{V}=-19.09\right)$, ESO 503-G007 $\left(M_{V}=-18.70\right)$, and ESO 438-G012 $\left(M_{V}=-18.67\right)$. These values are within 0.5 mag of the total magnitudes in the NED.

\subsection{Radial Number Density}

In Figure 4 we present the dwarf galaxy candidate number density as a function of the projected radius $(R)$ from the center of NGC 3585. The profile declines from the center, followed by flattening in the outermost region $\left(1.5 \lesssim R \lesssim 2^{\circ}\right)$. If the flattening represents the distribution of unrelated background galaxies, then the observed number density profile is compatible with a surface density distribution that is either exponential (Figure 4(a)) or follows a power law (Figure 4(b)). For the exponential form, the best-fit function is $\Sigma=$ $e^{-1.95( \pm 0.67) R+3.31( \pm 0.55)}+1.80( \pm 0.50) \quad \mathrm{deg}^{-2}$, while for the power-law form, it is $\Sigma=2.90( \pm 0.93) R^{-1.52( \pm 0.29)}+$ $1.80 \mathrm{deg}^{-2}$. Based on the former, we estimate the background contamination to be 10 objects ( $23 \%$ of our total) within a 

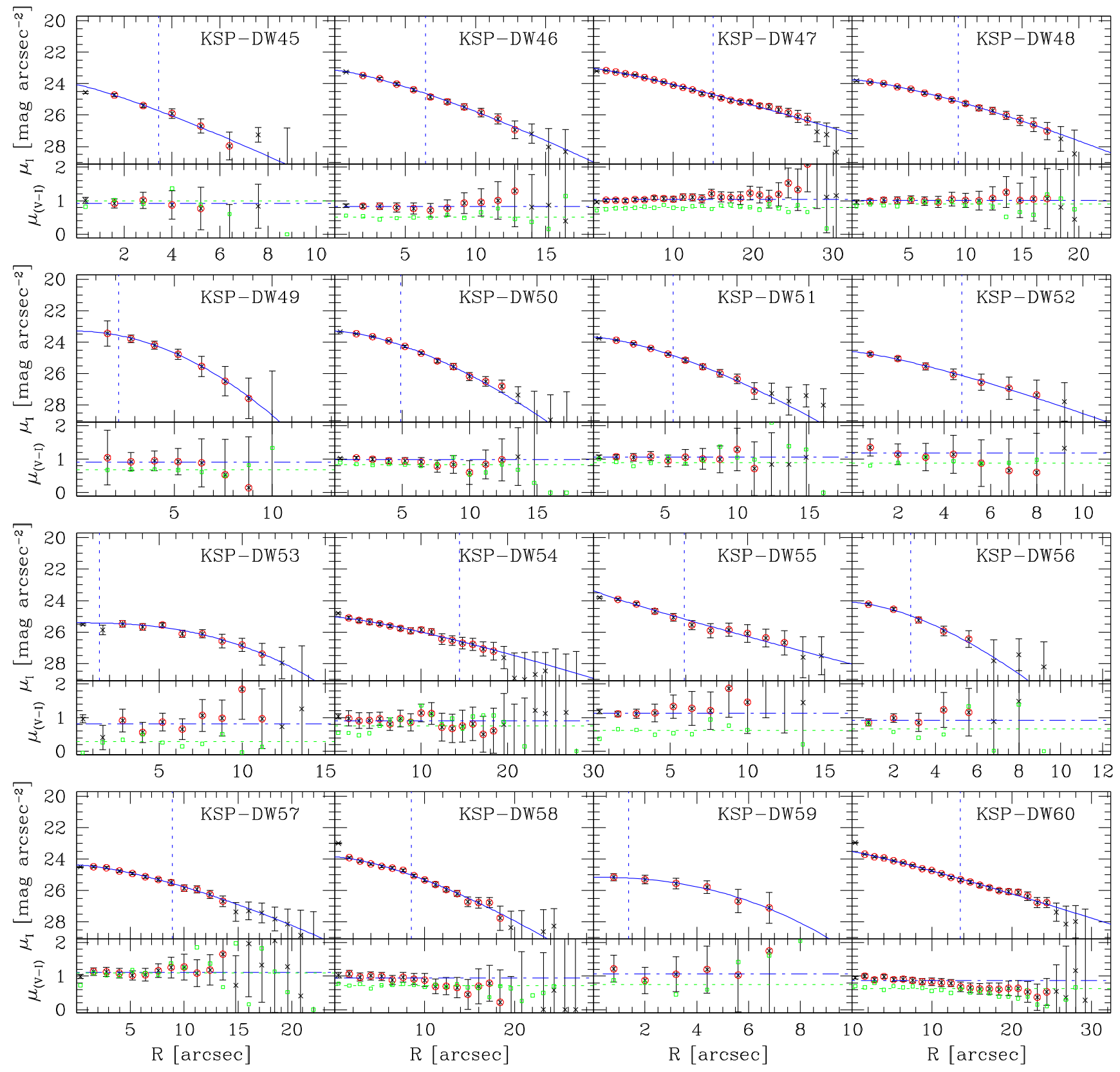

Figure 3. (Continued.)

1.5 radius of NGC 3585 . We believe that the remaining 4 dwarf galaxy candidates located beyond $R=1.5$ are most likely background sources. The contamination level we derive from the exponential model, $1.80 \mathrm{deg}^{-2}$, is consistent with the contamination value, $1.75 \mathrm{deg}^{-2}$, estimated from the background field counts (see Section 3.5 for details).

We considered the possibility of using SBF measurement to ascertain membership, but considering our observing conditions (1". 3 seeing in $I$ band), the target distance $(20 \mathrm{Mpc})$, and the exposure time $(10 \mathrm{hr})$. By comparing our results to some work in this area (e.g., Mieske et al. 2003; Carlsten et al. $2019 \mathrm{~b}$ ), we confirmed out expectation that this is not possible.

\subsection{Color and Structure Parameters}

We present the color-magnitude diagrams for the dwarf galaxy candidates in the NGC 3585 field in Figure 5. The mean
$(B-V)_{0}$ and $(V-I)_{0}$ of the dwarf galaxy candidates corrected for Galactic extinction are $0.69 \pm 0.18$ and $0.87 \pm 0.14$, respectively. These mean values are similar to those obtained for other groups: the M106 group, $\left\langle(B-V)_{0}\right\rangle \approx 0.73$ (Kim et al. 2011), the NGC 2784 group, $\left\langle(B-V)_{0}\right\rangle \approx 0.67$, $\left\langle(V-I)_{0}\right\rangle \approx 0.85$ (Park et al. 2017), and the M83 group, $\left\langle(B-V)_{0}\right\rangle \approx 0.82$ (Müller et al. 2015). When the colors of the dwarf galaxy candidates are overlaid with those of dwarf galaxies in other groups (Figure 5), we find that the color distributions of the NGC 3585 dwarf galaxy candidates are consistent with those of other groups (e.g., the M83 group from Müller et al. 2015 and the NGC 2784 group from Park et al. 2017). In general, the early-type galaxies in galaxy clusters have a color-magnitude relation: the brighter the galaxies, the redder they appear (e.g., the Virgo cluster from Lisker et al. 2008 and the Ursa Major cluster from Pak et al. 2014). The dwarf galaxy 

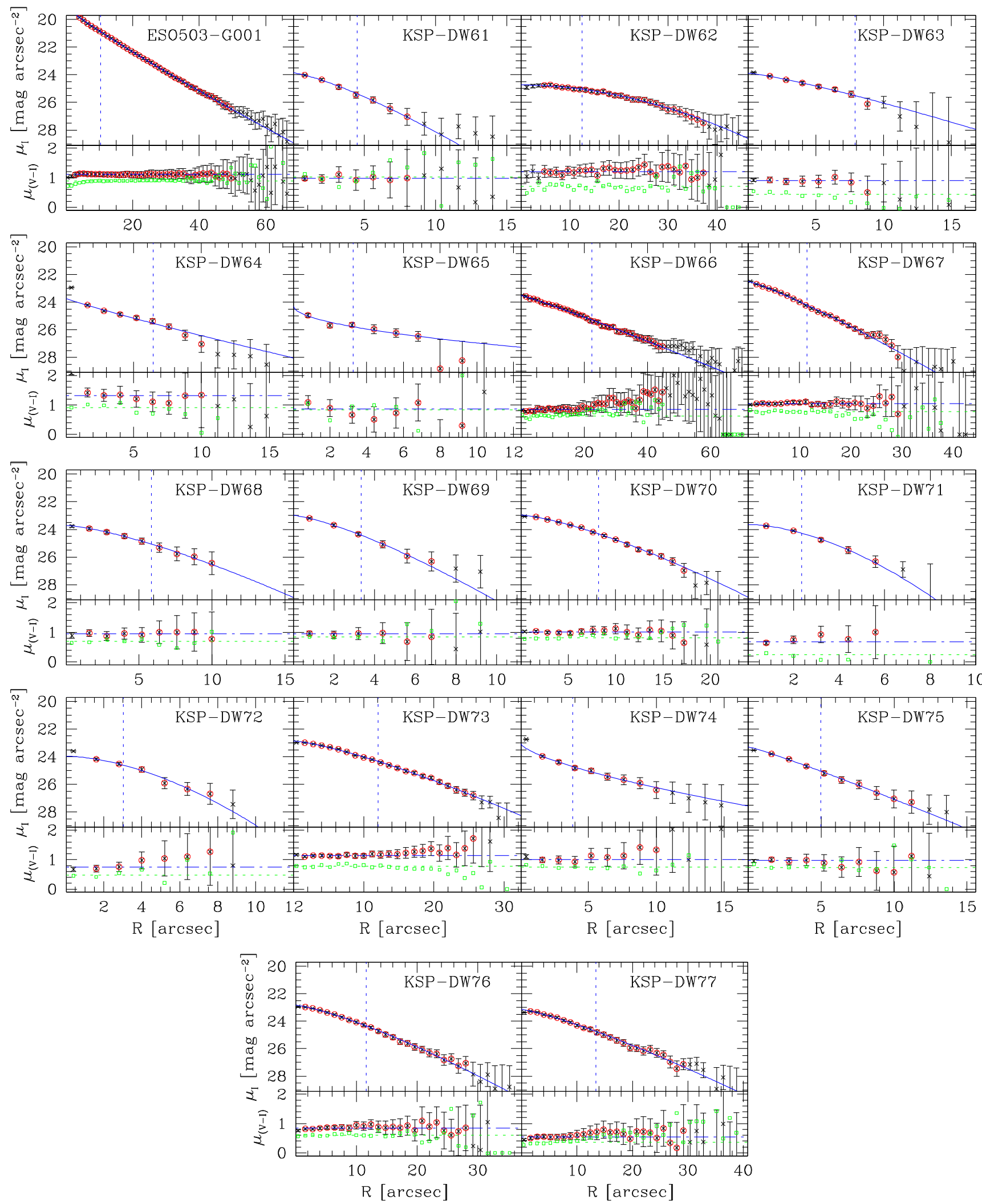

Figure 3. (Continued.)

candidates in the NGC 3585 also follow the relation, as shown in Figure 5. When we use dwarf galaxy candidates brighter than $M_{V}=-11 \mathrm{mag}$ in the NGC 3585 group from this study and the NGC 2784 group from Park et al. (2017), the dwarf galaxy candidates follow color-magnitude relations: $(B-V)_{0}=$ $-0.012 M_{V}+0.518$ and $(V-I)_{0}=-0.013 M_{V}+0.691$. 
Table 3

Photometric Catalog for Dwarf Galaxy Candidates in the NGC 3585 Field

\begin{tabular}{|c|c|c|c|c|c|c|c|c|c|c|}
\hline ID $^{a}$ & $\begin{array}{l}\text { R.A.(J2000) } \\
\text { (hh:mm:ss) }\end{array}$ & $\begin{array}{l}\text { Decl.(J2000) } \\
\text { (dd:mm:ss) }\end{array}$ & $\begin{array}{c}I^{\mathrm{b}} \\
(\mathrm{mag})\end{array}$ & $\begin{array}{c}(V-I)^{\mathrm{c}} \\
(\mathrm{mag})\end{array}$ & $\begin{array}{c}(B-V)^{\mathrm{c}} \\
(\mathrm{mag})\end{array}$ & $\begin{array}{c}\mu_{0, I}^{\mathrm{d}} \\
\left(\operatorname{mag} \operatorname{arcsec}^{-2}\right)\end{array}$ & $\begin{array}{c}r_{0, I}^{\mathrm{d}} \\
(\operatorname{arcsec})\end{array}$ & $n_{I}^{\mathrm{d}}$ & $\begin{array}{c}r_{e, I}^{\mathrm{d}} \\
(\operatorname{arcsec})\end{array}$ & Flag $^{\mathrm{e}}$ \\
\hline ESO502-G018 & 11:07:18.1 & $-25: 34: 23$ & 14.01 & 0.98 & 0.83 & $22.35 \pm 0.04$ & $23.57 \pm 0.68$ & $0.70 \pm 0.03$ & 25.72 & \\
\hline KSP-DW32 & 11:07:29.4 & $-25: 53: 50$ & 18.29 & 1.00 & 0.83 & $23.74 \pm 0.08$ & $5.85 \pm 0.50$ & $0.78 \pm 0.09$ & 7.71 & \\
\hline KSP-DW33 & 11:08:40.8 & $-25: 52: 02$ & 19.48 & 0.93 & 0.81 & $23.56 \pm 0.15$ & $3.40 \pm 0.40$ & $0.66 \pm 0.12$ & 3.39 & \\
\hline KSP-DW34 & 11:08:42.3 & $-25: 51: 26$ & 18.01 & 0.75 & 0.63 & $22.98 \pm 0.06$ & $5.36 \pm 0.27$ & $0.57 \pm 0.05$ & 3.78 & \\
\hline KSP-DW35 & 11:09:02.3 & $-25: 57: 16$ & 19.02 & 0.85 & 0.83 & $23.53 \pm 0.11$ & $4.12 \pm 0.38$ & $0.66 \pm 0.12$ & 4.12 & \\
\hline KSP-DW36 & $11: 10: 14.2$ & $-26: 27: 24$ & 20.13 & 0.90 & 0.47 & $23.65 \pm 0.08$ & $2.29 \pm 0.31$ & $0.85 \pm 0.20$ & 3.32 & \\
\hline KSP-DW37 & $11: 10: 36.1$ & $-26: 06: 59$ & 18.99 & 0.88 & 0.92 & $23.34 \pm 0.29$ & $2.88 \pm 0.84$ & $1.03 \pm 0.25$ & 4.90 & $\mathrm{~N}$ \\
\hline KSP-DW38 & $11: 10: 42.7$ & $-27: 01: 15$ & 20.17 & 0.80 & 0.82 & $23.69 \pm 0.05$ & $2.74 \pm 0.15$ & $0.58 \pm 0.09$ & 2.02 & \\
\hline KSP-DW39 & $11: 10: 46.2$ & $-26: 31: 31$ & 18.78 & 1.12 & 0.73 & $24.55 \pm 0.15$ & $7.65 \pm 0.91$ & $0.60 \pm 0.22$ & 6.21 & \\
\hline KSP-DW40 & $11: 10: 48.5$ & $-25: 15: 32$ & 16.38 & 0.92 & 0.63 & $21.94 \pm 0.04$ & $5.48 \pm 0.29$ & $0.93 \pm 0.04$ & 8.68 & \\
\hline GALEXASCJ & $11: 10: 51.5$ & $-25: 44: 58$ & 14.19 & 0.98 & 0.81 & $19.15 \pm 0.15$ & $2.29 \pm 0.31$ & $1.49 \pm 0.06$ & 4.40 & \\
\hline ESO438-G010 & 11:10:51.8 & $-27: 53: 51$ & 13.08 & 0.88 & 0.71 & $20.05 \pm 0.02$ & $8.72 \pm 0.19$ & $1.13 \pm 0.01$ & 15.62 & $\mathrm{~N}$ \\
\hline KSP-DW41 & $11: 11: 24.1$ & $-27: 58: 09$ & 18.35 & 0.85 & 0.73 & $23.52 \pm 0.12$ & $5.34 \pm 0.54$ & $0.73 \pm 0.11$ & 6.39 & $\mathrm{~N}$ \\
\hline KSP-DW42 & $11: 11: 26.1$ & $-26: 32: 54$ & 18.14 & 0.96 & 0.87 & $24.89 \pm 0.08$ & $12.65 \pm 0.63$ & $0.49 \pm 0.07$ & 5.24 & \\
\hline KSP-DW43 & $11: 11: 44.4$ & $-26: 48: 38$ & 18.60 & 0.84 & 0.95 & $24.83 \pm 0.11$ & $9.40 \pm 0.77$ & $0.60 \pm 0.15$ & 7.63 & \\
\hline KSP-DW44 & $11: 11: 57.2$ & $-26: 54: 59$ & 15.16 & 1.05 & 0.95 & $21.99 \pm 0.03$ & $9.92 \pm 0.33$ & $0.92 \pm 0.03$ & 15.58 & $\mathrm{~N}$ \\
\hline KSP-DW45 & $11: 12: 02.7$ & $-26: 25: 44$ & 20.45 & 0.92 & 1.00 & $24.07 \pm 0.78$ & $2.42 \pm 1.55$ & $0.84 \pm 0.59$ & 3.44 & \\
\hline KSP-DW46 & $11: 12: 13.3$ & $-27: 14: 17$ & 18.03 & 0.83 & 0.51 & $23.14 \pm 0.16$ & $5.09 \pm 0.75$ & $0.76 \pm 0.14$ & 6.45 & \\
\hline KSP-DW47 & $11: 12: 16.3$ & $-26: 11: 16$ & 16.23 & 1.05 & 0.80 & $23.03 \pm 0.04$ & $10.46 \pm 0.37$ & $0.84 \pm 0.04$ & 14.99 & \\
\hline KSP-DW48 & $11: 12: 23.4$ & $-25: 50: 52$ & 17.75 & 1.01 & 0.90 & $23.75 \pm 0.07$ & $7.80 \pm 0.52$ & $0.74 \pm 0.08$ & 9.38 & \\
\hline KSP-DW49 & $11: 12: 34.8$ & $-28: 01: 43$ & 18.82 & 0.99 & 0.68 & $23.30 \pm 0.12$ & $4.41 \pm 0.34$ & $0.51 \pm 0.09$ & 2.18 & \\
\hline KSP-DW50 & $11: 12: 51.6$ & $-27: 07: 33$ & 18.19 & 0.98 & 0.84 & $23.30 \pm 0.06$ & $5.55 \pm 0.28$ & $0.63 \pm 0.06$ & 4.89 & \\
\hline KSP-DW51 & $11: 12: 53.2$ & $-26: 29: 03$ & 18.62 & 1.06 & 0.90 & $23.68 \pm 0.10$ & $5.24 \pm 0.42$ & $0.68 \pm 0.09$ & 5.56 & \\
\hline KSP-DW52 & $11: 12: 56.2$ & $-27: 26: 41$ & 20.18 & 1.16 & 0.87 & $24.60 \pm 0.18$ & $3.67 \pm 0.71$ & $0.78 \pm 0.25$ & 4.77 & \\
\hline KSP-DW53 & $11: 12: 56.8$ & $-26: 39: 55$ & 19.51 & 0.82 & 0.29 & $25.41 \pm 0.30$ & $8.82 \pm 1.32$ & $0.40 \pm 0.27$ & 1.39 & \\
\hline KSP-DW54 & 11:13:01.0 & $-26: 51: 23$ & 18.23 & 0.91 & 0.80 & $25.04 \pm 0.20$ & $11.00 \pm 1.83$ & $0.78 \pm 0.26$ & 14.42 & $\mathrm{U}$ \\
\hline KSP-DW55 & 11:13:09.0 & $-26: 19: 56$ & 18.52 & 1.13 & 0.64 & $23.35 \pm 0.28$ & $3.33 \pm 1.13$ & $1.11 \pm 0.30$ & 5.90 & \\
\hline KSP-DW56 & $11: 13: 27.9$ & $-26: 56: 07$ & 20.15 & 0.91 & 0.67 & $24.11 \pm 0.12$ & $3.29 \pm 0.36$ & $0.62 \pm 0.16$ & 2.83 & \\
\hline KSP-DW57 & $11: 13: 32.6$ & $-26: 32: 48$ & 18.29 & 1.11 & 1.09 & $24.37 \pm 0.12$ & $8.40 \pm 0.79$ & $0.68 \pm 0.14$ & 8.92 & \\
\hline KSP-DW58 & $11: 13: 36.3$ & $-26: 33: 49$ & 17.85 & 0.91 & 0.71 & $23.86 \pm 0.08$ & $8.16 \pm 0.57$ & $0.68 \pm 0.07$ & 8.50 & $\mathrm{~N}$ \\
\hline KSP-DW59 & $11: 13: 48.1$ & $-26: 07: 58$ & 20.38 & 1.07 & 0.75 & $25.15 \pm 0.17$ & $5.17 \pm 0.66$ & $0.44 \pm 0.22$ & 1.38 & \\
\hline KSP-DW60 & 11:13:55.2 & $-26: 22: 20$ & 16.98 & 0.86 & 0.61 & $23.52 \pm 0.06$ & $8.92 \pm 0.54$ & $0.89 \pm 0.06$ & 13.56 & $\mathrm{~N}, \mathrm{U}$ \\
\hline ESO503-G001 & $11: 14: 02.2$ & $-26: 21: 56$ & 13.00 & 1.11 & 0.90 & $19.08 \pm 0.06$ & $5.87 \pm 0.27$ & $1.11 \pm 0.03$ & 10.43 & \\
\hline KSP-DW61 & $11: 14: 13.1$ & $-26: 31: 38$ & 19.57 & 0.98 & 1.05 & $23.86 \pm 0.13$ & $3.47 \pm 0.52$ & $0.77 \pm 0.18$ & 4.47 & \\
\hline KSP-DW62 & $11: 14: 14.4$ & $-26: 30: 47$ & 16.57 & 1.21 & 0.71 & $24.74 \pm 0.07$ & $23.93 \pm 1.09$ & $0.52 \pm 0.07$ & 12.45 & $\mathrm{U}$ \\
\hline KSP-DW63 & 11:14:16.9 & $-27: 04: 44$ & 18.44 & 0.90 & 0.45 & $23.87 \pm 0.27$ & $5.64 \pm 1.29$ & $0.83 \pm 0.40$ & 7.90 & \\
\hline KSP-DW64 & $11: 14: 17.2$ & $-26: 29: 51$ & 18.69 & 1.31 & 0.91 & $23.72 \pm 0.41$ & $3.63 \pm 1.67$ & $1.11 \pm 0.47$ & 6.44 & $\mathrm{~N}$ \\
\hline KSP-DW65 & $11: 14: 18.9$ & $-27: 20: 56$ & 18.33 & 0.86 & 0.83 & $24.27 \pm 0.85$ & $1.70 \pm 2.30$ & $1.95 \pm 1.55$ & 3.27 & \\
\hline KSP-DW66 & $11: 14: 27.9$ & $-26: 55: 00$ & 15.89 & 0.86 & 0.64 & $23.52 \pm 0.05$ & $14.32 \pm 0.82$ & $0.92 \pm 0.06$ & 22.47 & $\mathrm{U}$ \\
\hline KSP-DW67 & $11: 14: 29.6$ & $-25: 16: 10$ & 16.32 & 1.05 & 0.77 & $22.51 \pm 0.04$ & $7.79 \pm 0.27$ & $0.86 \pm 0.03$ & 11.43 & \\
\hline KSP-DW68 & $11: 14: 36.4$ & $-26: 51: 15$ & 18.70 & 0.94 & 0.70 & $23.71 \pm 0.16$ & $4.98 \pm 0.65$ & $0.73 \pm 0.16$ & 5.85 & \\
\hline KSP-DW69 & 11:14:54.3 & $-27: 49: 00$ & 19.26 & 0.95 & 0.85 & $22.98 \pm 0.09$ & $2.71 \pm 0.25$ & $0.75 \pm 0.10$ & 3.34 & \\
\hline KSP-DW70 & $11: 15: 24.1$ & $-26: 45: 14$ & 17.23 & 1.01 & 0.82 & $22.96 \pm 0.04$ & $6.95 \pm 0.28$ & $0.73 \pm 0.04$ & 8.21 & \\
\hline KSP-DW71 & 11:15:58.1 & $-27: 29: 57$ & 19.76 & 0.68 & 0.25 & $23.65 \pm 0.08$ & $3.24 \pm 0.25$ & $0.58 \pm 0.12$ & 2.35 & \\
\hline KSP-DW72 & $11: 16: 21.2$ & $-26: 24: 26$ & 19.56 & 0.75 & 0.47 & $23.96 \pm 0.21$ & $4.10 \pm 0.65$ & $0.58 \pm 0.18$ & 3.04 & \\
\hline KSP-DW73 & $11: 16: 47.3$ & $-27: 40: 17$ & 16.39 & 1.14 & 0.78 & $22.86 \pm 0.03$ & $9.52 \pm 0.27$ & $0.76 \pm 0.03$ & 12.07 & \\
\hline KSP-DW74 & $11: 17: 55.0$ & $-26: 07: 35$ & 18.37 & 1.01 & 0.75 & $23.03 \pm 0.75$ & $1.99 \pm 2.03$ & $1.49 \pm 0.82$ & 3.83 & $\mathrm{~N}$ \\
\hline KSP-DW75 & $11: 18: 26.3$ & $-26: 41: 52$ & 18.93 & 0.97 & 0.74 & $23.29 \pm 0.20$ & $3.21 \pm 0.62$ & $0.91 \pm 0.16$ & 4.97 & \\
\hline KSP-DW76 & $11: 18: 26.4$ & $-26: 11: 39$ & 16.46 & 0.84 & 0.60 & $22.83 \pm 0.03$ & $9.02 \pm 0.30$ & $0.77 \pm 0.03$ & 11.62 & \\
\hline KSP-DW77 & 11:18:37.1 & $-26: 55: 09$ & 16.53 & 0.55 & 0.37 & $23.14 \pm 0.05$ & $9.81 \pm 0.57$ & $0.81 \pm 0.06$ & 13.45 & \\
\hline
\end{tabular}

Notes.

${ }^{a} \mathrm{KSP} \mathrm{DW}{ }^{* *}$ candidates are newly discovered objects in KSP. The full name of GALEXASCJ candidate is GALEXASCJ111051.

${ }^{b} I$ is the total $I$-band magnitude derived from the Sérsic fit.

c $(V-I)$ and $(B-V)$ are colors without correction for extinction.

d $\mu_{0, I}, r_{0, I}, n_{I}$, and $r_{e, I}$ are central surface brightness, scale length, curvature index, and effective radius derived for the $I$-band Sérsic fits, respectively.

${ }^{\mathrm{e}} \mathrm{N}$ and $\mathrm{U}$ flag a nucleated galaxy candidate and UDG candidate, respectively.

These results for the color distribution and relation indicate that many of the dwarf galaxy candidates in the NGC 3585 field may be early-type galaxies.
In Figure 6 we compare the structural parameters, $V$-band central surface brightnesses $\left(\mu_{0}\right)$, effective radii $\left(r_{e}\right)$, and Sérsic$n$ indices of the dwarf galaxy candidates in the NGC 3585 field 


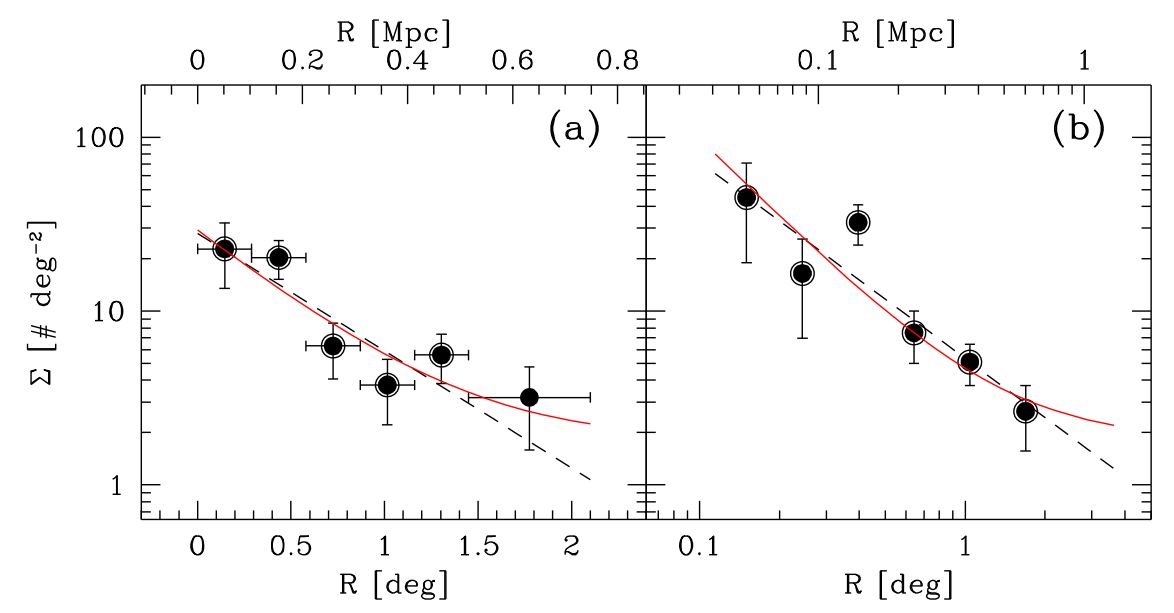

Figure 4. Radial projected number density of the dwarf galaxy candidates in the NGC 3585 field. (a) The radial binning is done in equal linear intervals except for the last bin. The dashed line and the solid curve represent the best-fit exponential function for $R \lesssim 1.5$ and the exponential + constant function, respectively. (b) The radial binning is done in equal logarithmic intervals, except for the last bin. The black dashed line and the red solid curve represent the best-fit power-law functions without and with an added constant value, respectively.

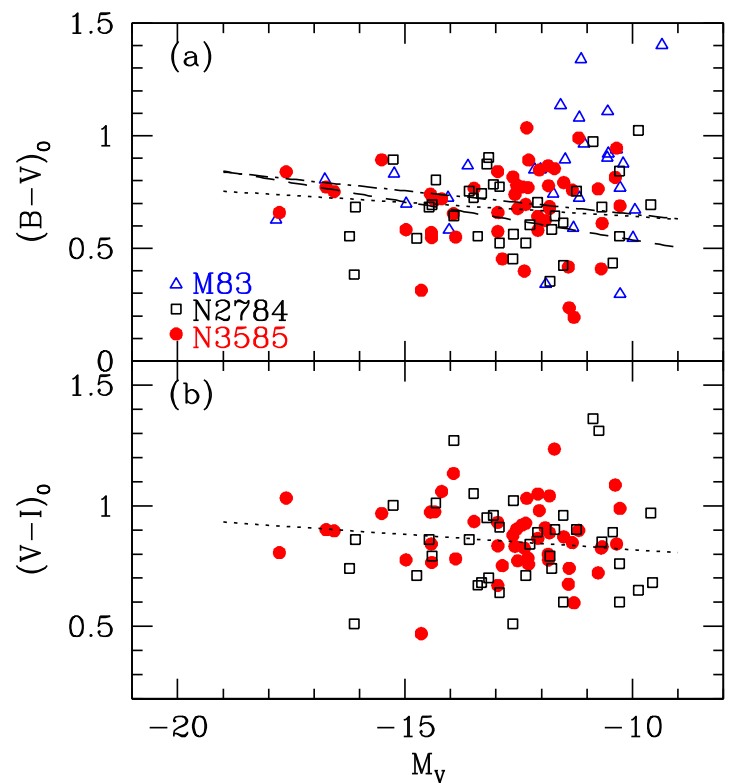

Figure 5. Color-magnitude diagrams of the dwarf galaxy candidates in the NGC 3585 fields. The circles, squares, and triangles represent the dwarf galaxy candidates in the NGC 3585 group (this study), NGC 2784 group (Park et al. 2017), and the M83 group (Müller et al. 2015), respectively. The dotted lines indicate the color-magnitude relations obtained from the dwarf galaxy candidates in the NGC 2784 group and the NGC 3585 group, while the dotdashed and dashed lines are obtained from the early-type galaxies in the Virgo cluster (Lisker et al. 2008) and the Ursa Major cluster (Pak et al. 2014), respectively.

to those found in other groups. The $\mu_{0, V}$ and $M_{V}$ values are derived from the estimated $\mu_{0, I}$ and $M_{I}$ (Section 3.2) using the $(V-I)$ color of the dwarf galaxy candidates assuming that they are at the same distance as NGC 3585. The $r_{e}$ and $n$ values are those obtained from fits to the $I$-band images. The values for the structural parameters of dwarf galaxies in other groups are compiled from the literature: Müller et al. (2015) for the M83 group, Chiboucas et al. (2009) for the M81 group, and Park et al. (2017) for the NGC 2784 group. As seen in the M83 group (Müller et al. 2015) and in the NGC 2784 group (Park et al. 2017), the central surface brightnesses and the effective radii of the dwarf galaxy candidates in the NGC 3585 group increase with galaxy luminosity. In the case of the Sérsic

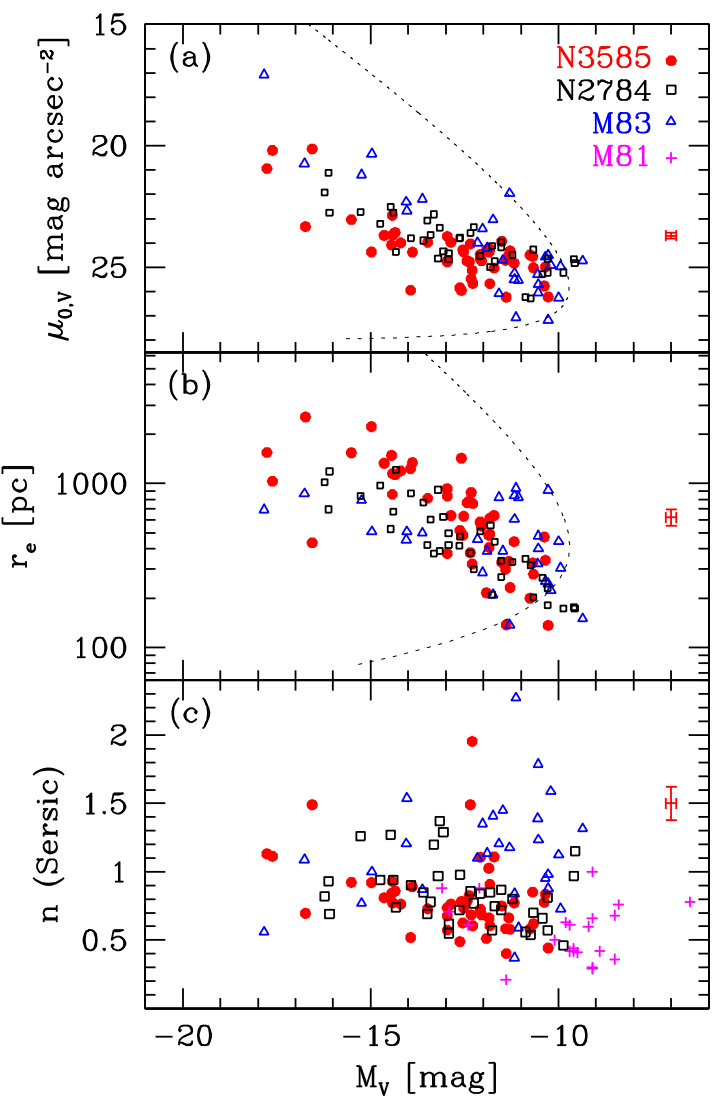

Figure 6. Central surface brightness (a), effective radius (b), and Sérsic-n (c) vs. total absolute magnitude for the dwarf galaxy candidates. The circles, squares, triangles, and pluses indicate the dwarf galaxy candidates in the NGC 3585 group (this study), NGC 2784 group (Park et al. 2017), M83 group (Müller et al. 2015), and M81 group (Chiboucas et al. 2009), respectively. The dotted curves represent the completeness limits for our survey (see the Section 3.1). The error bar in each panel represents the mean value of the uncertainties in each parameter.

curvature index, the median value, $n \approx 0.8 \pm 0.1$, of the dwarf galaxy candidates in the NGC 3585 field is similar to that of dwarf galaxies in other groups: $n \approx 1.1 \pm 0.3$ for the M83 group (Müller et al. 2015), $n \approx 0.6 \pm 0.2$ for the M81 group (Chiboucas et al. 2009), and $n \approx 0.8 \pm 0.1$ for the NGC 2784 
group (Park et al. 2017). The properties of the NGC 3585 dwarf galaxy candidates are consistent with those of dwarf galaxies in other groups.

\subsection{Estimation of the Contamination Level by Background Diffuse Sources}

In order to estimate the level of contamination of the dwarf galaxy candidates in NGC 3585 fields identified in Section 3.1 by sources in background, we adopt the KK196 field as a control field. The KK 196 field (R.A.J2000 $=13^{\mathrm{h}} 21^{\mathrm{m}} 47^{\mathrm{s}} \cdot 42$,

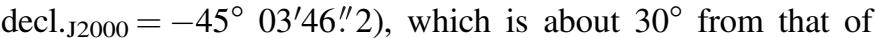
NGC 3585, has been observed together with the NGC 3585 field in our KSP program, so that both the fields have almost identical integrated exposure times and depth in stacked images. In addition, the KK 196 field belongs to the Centaurus A group at $4 \mathrm{Mpc}$ distance, and this makes the use of the field as a control field more reliable because the dwarf galaxies in this field associated with the Centaurus A group can easily be resolved due to their proximity. Our three classifiers searched for dwarf galaxy candidates in the KK196 field in the same manner as described in Section 3.1 As a result, we found four resolved dwarf galaxy candidates that belong to the Centaurus A group - two of them were previously identified as KK196 and KK203, while the rest two are newly indentified in this study-as well as seven unresolved dwarf galaxy candidadtes that appear to be unassociated with the Centaurus A group. We therefore consider these seven candidates as background dwarf galaxies.

We now carry out the same analysis on these seven candidates as we did on the NGC 3585 dwarf galaxy candidates. Results will be reported in greater detail by $\mathrm{H}$. S. Park et al. (2019, in preparation). We summarize our results with a focus on understanding the background contamination in the NGC 3585 field as follows: (1) the spatial distribution of the seven observed diffuse candidates is compatible with a random distribution from both the $1 \mathrm{D}$ and 2D KolmogorovSmirnov tests (Press \& Teukolsky 1998), (2) assuming that these objects are at the distance of NGC 3585, their distribution in the color-magnitude diagram (mean colors $(B-V)_{0}=$ $0.67 \pm 0.08$ and $(V-I)_{0}=0.82 \pm 0.10$ and $-13.6<M_{V}<$ $-11.1 \mathrm{mag}$ ) and their Sérsic structural parameters (median $n \approx 0.7 \pm 0.2$,) are consistent with those of the NGC 3585 dwarf galaxy candidates, (3) their projected number density is about $7 /\left(2^{\circ} \times 2^{\circ}\right)$, or $1.75( \pm 0.66) \mathrm{deg}^{-2}$, which is very similar to the value estimated from the number density profile of NGC 3585 dwarf galaxy candidates (1.80 deg $\mathrm{de}^{-2}$, see Section 3.3), and (4) none of these candidates have either a nuclear source or are UDGs. While the color and magnitude similarities make it difficult to reject this type of background source, we have a robust estimate of their contribution to the satellite number density profiles and an indication that one way to identify bona fide dwarf galaxies may be if they are either nucleated (see Section 4.2) or ultra diffuse (see Section 4.3).

\section{Nature of the NGC 3585 Group}

\subsection{Radial Distributions}

In clusters and groups, the more massive galaxies tend to be centrally concentrated (Presotto et al. 2012; Roberts et al. 2015). To investigate if dwarf galaxies in the NGC 3585 group conform to this trend, we divide our sample into two: bright $\left(M_{V}<-12.5 \mathrm{mag}\right)$ and faint $\left(M_{V} \geqslant-12.5 \mathrm{mag}\right)$ objects. We

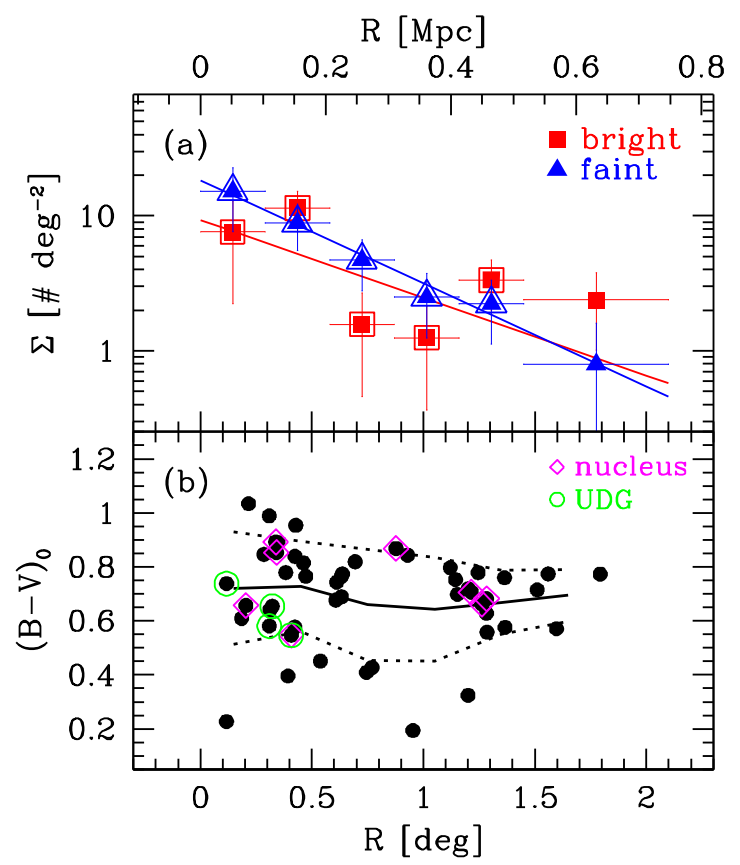

Figure 7. Radial number density profile (a) and color distribution (b) for the dwarf galaxy candidates in the NGC 3585 group. The squares and triangles in (a) are the number densities for the bright $\left(M_{V}<-12.5\right)$ and faint $\left(M_{V} \geqslant-12.5\right)$ dwarf samples, respectively. The solid lines represent exponential fits to each sample. Only the measurements represented by larger open symbols are used in the fitting. The solid and dotted curves in (b) indicate the mean color value and standard deviation in each radial bin, respectively. The green open circles and magenta open diamonds represent the UDG and nucleated dwarf galaxy candidates in the NGC 3585 group, respectively.

show the radial number density profiles for these two subsamples in Figure 7(a). The best-fit exponential functions for the bright and faint populations are $\ln (\Sigma) \simeq-0.58$ $( \pm 0.41) \quad R+0.97 \quad( \pm 0.34)$ and $\ln (\Sigma) \simeq-0.76 \quad( \pm 0.08)$ $R+1.26( \pm 0.07)$, respectively. Given the uncertainties in the fits, both populations of dwarf galaxy candidates show similar density profiles. Thus, mass segregation is not apparent among the dwarf galaxy candidates in the NGC 3585 group. In Figure $7(\mathrm{~b})$ we plot the mean color of the dwarf galaxies in NGC 3585 versus projected radius from the group center. There is no apparent color variation with radius.

The mass and color radial behaviors of the dwarf galaxy candidates in the NGC 3585 differ from the significant radial dependencies that we found in the NGC 2784 group (Park et al. 2017). This difference suggests that the NGC 3585 group might be a dynamically younger system where mass segregation has not yet developed.

\subsection{Nucleated Dwarf Galaxies}

Some dwarf galaxies have a distinct nucleated source in their central region (Côté et al. 2006; Trentham \& Tully 2009; Turner et al. 2012) and the dwarf galaxy candidates in the NGC 3585 group are no exception. In Table 3 we classify 8 dwarf galaxy candidates among the 50 in the NGC 3585 group as nucleated based on the images (Figure 2) and the surface brightness profiles (Figure 3). These classified nucleated candidates have an evident point source that is distinctly brighter (approximately $\gtrsim 0.5 \mathrm{mag} \operatorname{arcsec}^{-2}$ ) than the central surface brightness estimated by Sérsic fit to the entire galaxy. The incidence of the nucleated dwarf galaxy candidates is $20 \%$ (8 out of 50) in the NGC 3585 group, which is consistent with 

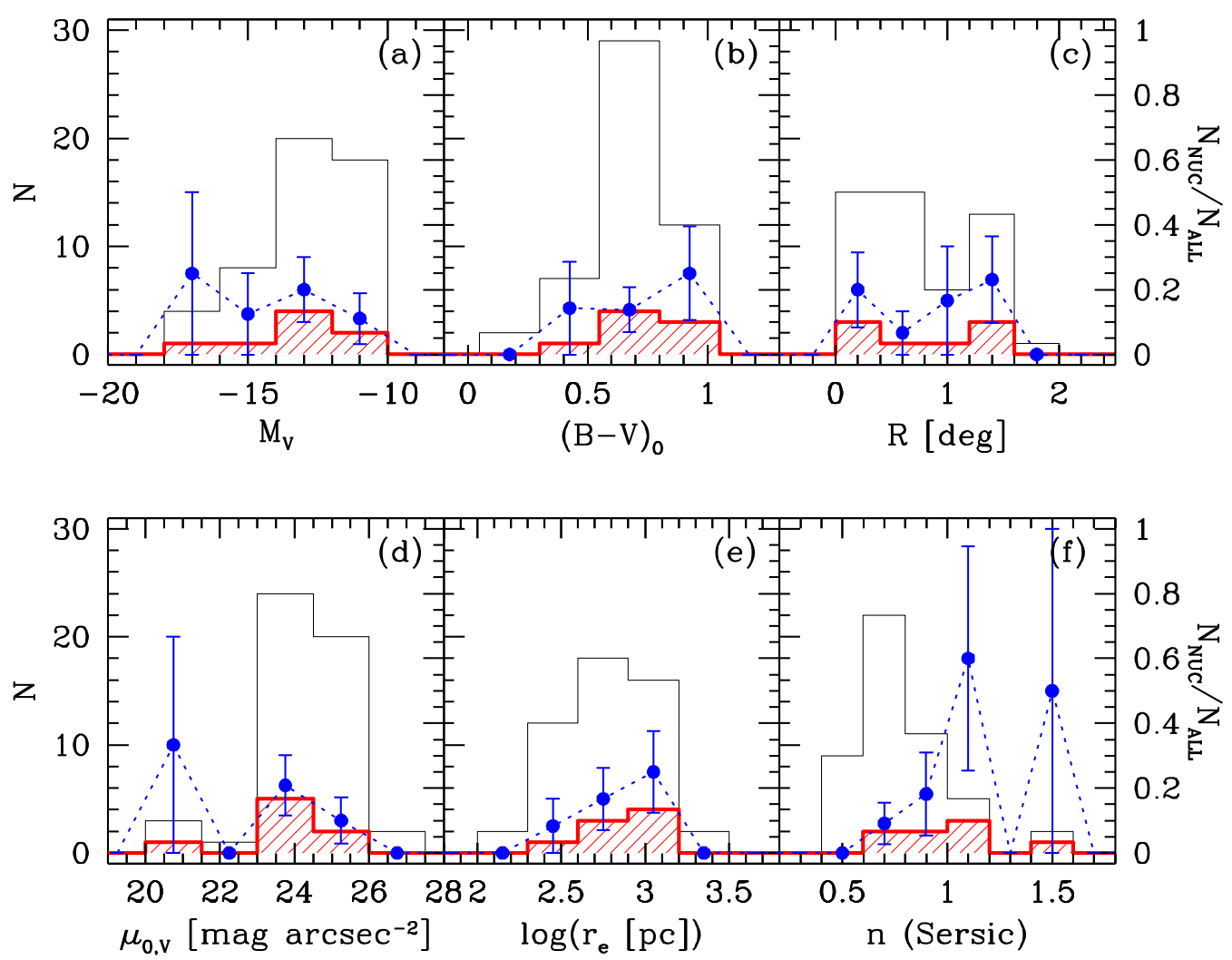

Figure 8. Number and number ratio distributions of nucleated dwarf galaxy candidates in terms of luminosity (a), color (b), projected distance from the group center (c), central surface brightness (d), log effective radius (e), and Sérsic-n (f). The solid and hatched histograms represent all the dwarf galaxy candidates and the nucleated dwarf galaxy candidates in the NGC 3585 group, respectively. The dotted curves with the filled circles indicate the fraction of nucleated dwarf galaxy candidates $\left(N_{\mathrm{NUC}} / N_{\mathrm{ALL}}\right)$, and error bars represent Poisson uncertainties.

that found in other groups (20\% in the LG early-type, Turner et al. 2012, and $10 \%-30 \%$ for several nearby galaxy groups, Trentham \& Tully 2009), and $10 \%$ in the NGC 2784 group $\left(\right.$ Park et al. 2017) ${ }^{8}$ ). In contrast, in the Virgo and Fornax clusters, studies have found that about $60 \%-80 \%$ of the dwarf galaxies have nuclei (Côté et al. 2006; Lisker et al. 2007; Turner et al. 2012; Georgiev \& Böker 2014). Although selection effects could play a role, cluster samples are biased to brighter samples; even our bright group sample has a small nucleated incidence ( $30 \%$; Figure 8(a)). Unfortunately, the significance of the group results is undermined by small number statistics. Confirmation of the difference between groups and clusters awaits larger samples. Nevertheless, none of the 7 dwarf galaxy candidates in the control field (see Section 3.5) has a nucleus, which indirectly supports a conjecture that the nucleated dwarf galaxy candidates are associated with the NGC 3585 group.

In Figure 8 we compare the luminosities $\left(M_{V}\right)$, colors $\left((B-V)_{0}\right)$, radial distribution from the group center $(R)$, central surface brightness $\left(\mu_{0, V}\right)$, effective radii $\left(r_{e}\right)$, and Sérsic- $n$ of the nucleated dwarf galaxy candidates to those of other dwarf galaxy candidates. We overlay the distribution of the number ratio $\left(N_{\mathrm{NUC}} / N_{\mathrm{ALL}}\right)$ in each panel. From these plots, we conclude that nucleated dwarf galaxy candidates are more common among brighter (a, d), redder (b), larger (e), and more centrally concentrated (f) dwarf galaxy candidates. We note that due to the relationships among these parameters, a

\footnotetext{
8 There are four nucleated dwarf galaxy candidates (KSP-DW13, NGC 2784 dw01, KK72, and KSP-DW15) in the NGC 2784 group.
}

high incidence for redder dwarf galaxy candidates is related in corresponding higher incidence among brighter, more concentrated dwarf galaxy candidates due to the color-magnitude relation and the magnitude-structural parameter trends described in Section 3.4 The correlation between nuclear sources and color is consistent with those obtained for dwarf elliptical galaxies (dEs) in nearby galaxy clusters (Rakos \& Schombert 2004; Lisker et al. 2007). However, the incidence of nucleated dwarf galaxy candidates does not vary with distance from the center in the NGC 3585 group (Figure 8(c)), which is in contrast to the trend in clusters. The nucleated dwarfs in the Virgo cluster are located in denser environments. If the radial trend in Virgo is due to mass segregation, with the nucleated dwarfs being the more massive and sinking toward the center, our result might again suggest that the NGC 3585 system is dynamically young.

\subsection{Ultra-diffuse Galaxies}

Numerous UDGs have recently been found in nearby galaxy clusters and groups (van Dokkum et al. 2015; van der Burg et al. 2016; Müller et al. 2018; Zaritsky et al. 2019). We find four UDG candidates in the NGC 3585 group based on central surface brightness $\left(\mu_{0, V} \gtrsim 23.7 \mathrm{mag} \operatorname{arcsec}^{-2}\right)$ and effective radius $\left(r_{e, V} \gtrsim 1.5 \mathrm{kpc}\right)$ criteria (van Dokkum et al. 2015). The UDG candidates in the NGC 3585 group (see Table 3 ) 9 have

\footnotetext{
9 The NGC 2784 group (Park et al. 2017) has just one UDG candidate (NGC $2784 \mathrm{dw} 01)$ located near the group center $(R=0.01 \mathrm{Mpc})$ with $\mu_{0, V}=$ $24.4 \mathrm{mag} \operatorname{arcsec}^{-2}$ and $(B-V)_{0}=0.8$, but the candidate can only marginally be classified as a UDG due to its small effective size of $r_{e, V}=1.3 \mathrm{kpc}$.
} 

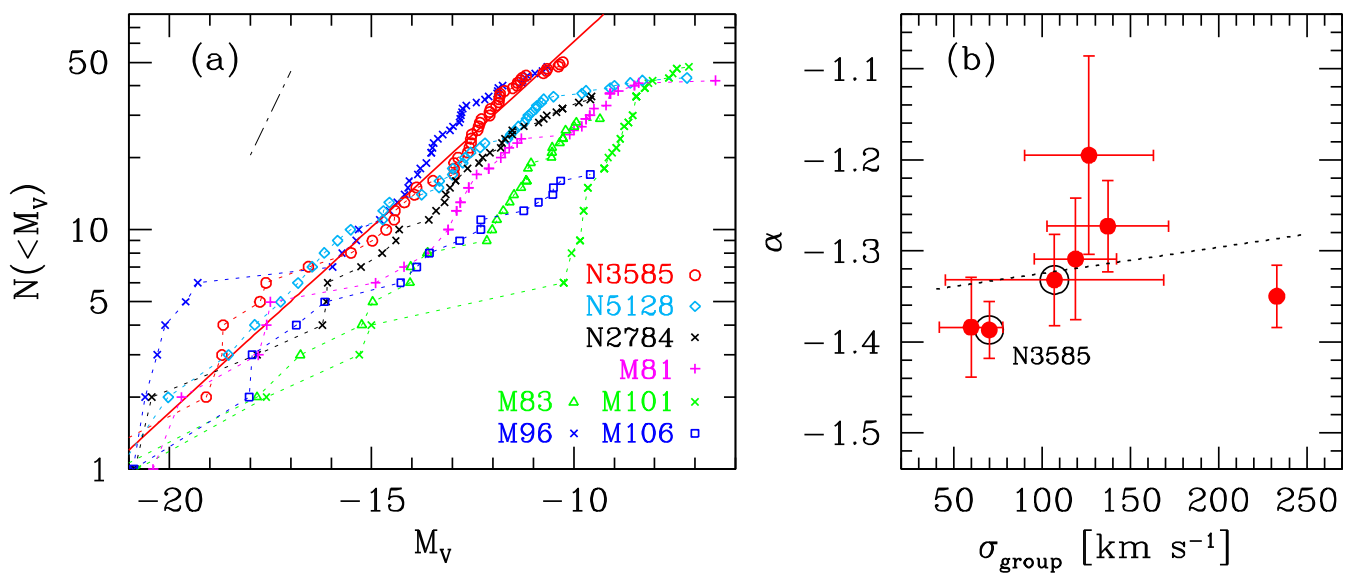

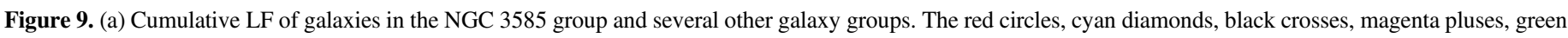

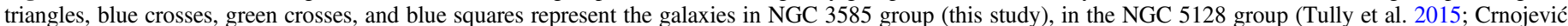

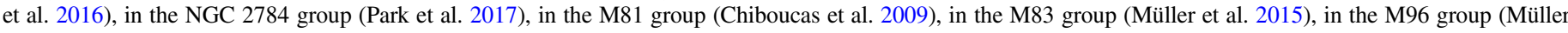

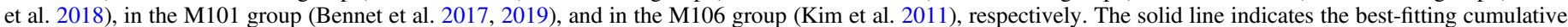

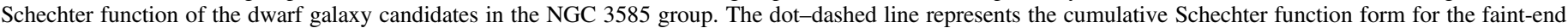

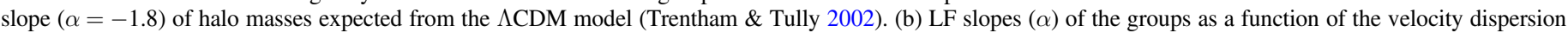

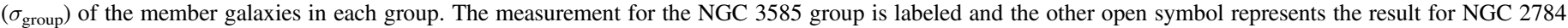

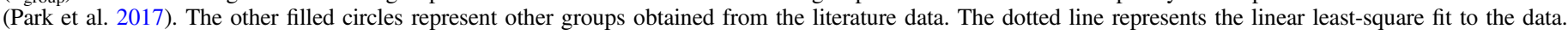

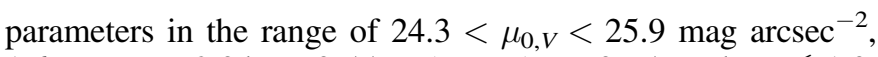
$1.6<r_{e, V}<2.0 \mathrm{kpc}$. $0.55 \leqslant(B-V)_{0} \leqslant 0.75$, and $n_{V} \lesssim 1.0$. The latter two quantities are similar to the average values, $(B-V)_{0} \approx 0.69$ and $n \approx 0.8$, of all the dwarf galaxy candidates in the NGC 3585 group. The absence of UDGs among the control field dwarf candidates (see Section 3.5) indirectly supports the conjecture that the UDG candidates are associated with the NGC 3585 group.

An interesting properties of the four UDGs in this group is that they are centrally concentrated, all within $R<0.15 \mathrm{Mpc}$ (Figure 7(b)). A strong central concentration is in conflict with results from UDGs in galaxy clusters (van Dokkum et al. 2015; van der Burg et al. 2016; Román \& Trujillo 2017a) and some galaxy groups (Merritt et al. 2016; Román \& Trujillo 2017b; Müller et al. 2018), which instead find a deficit of UDGs in the central region. While the statistical significance of the result for NGC 3585 is again limited due to small numbers, the result again suggests that the NGC 3585 group might be a dynamically younger system in which centrally located UDG candidates have not yet been tidally disrupted.

Finally, we add our measurement to previous measurements to investigate the number abundance of UDGs as a function of group mass. In this instance, we consider a velocity dispersion as a system mass. A number of four UDGs in a system with a velocity dispersion of $\sigma_{v}=70 \mathrm{~km} \mathrm{~s}^{-1}$ (see Section 3.1) causes a flattening of the relationship between the UDG number abundance and system mass (see Figure 6 by Román \& Trujillo 2017 b or Figure 10 by Lee et al. 2017). If confirmed for similar low-mass groups, this result may indicate that UDGs form preferentially in groups (Román \& Trujillo 2017b) or are less effectively destroyed.

\subsection{Comparison with Luminosity Functions of Other Groups}

To compare the LF of the galaxies in the NGC 3585 group with those of other galaxy groups, we use cumulative LFs because, in general, the number of galaxies in a group is small (Trentham \& Tully 2009). To construct the LF for the NGC 3585 group, we only use galaxies with $R<0.5 \mathrm{Mpc}$ to minimize the effect of background contamination (Section 3.3).
The other groups in this comparison include the M81 group (Chiboucas et al. 2009), the M83 group (Müller et al. 2015), the M96 group (Müller et al. 2018), the M101 group (Bennet et al. 2017, 2019; Danieli et al. 2017; Carlsten et al. 2019a), the M106 group (Kim et al. 2011), the NGC 2784 group (Park et al. 2017), and the NGC 5128 group (Tully et al. 2015; Crnojević et al. 2016). The $R<0.5 \mathrm{Mpc}$ condition is satisfied for all of these other groups except for the NGC 5128 group. However, in this case, most of the dwarfs are spectroscopically confirmed members, therefore contamination is not an issue. In Figure 9(a) we compare the cumulative LFs of the NGC 3585 group to those of these seven other groups.

The eight groups have roughly similar LF shapes and faintend slopes. However, we do note that certain groups (M81, M83, NGC 2784, and NGC 3585) have a clear faint-end hump, a steep increase in the cumulative numbers around $M_{V} \approx-13$ mag. These LF humps could be a real structure, with physical meaning that could be exploited, or perhaps they are the result of background contamination. To resolve this ambiguity, either deep spectroscopic surveys to determine membership are required or a larger, homogeneous photometric survey, such as our complete KMTNet dwarf galaxy search project. With a much larger sample it would be possible to establish whether this is a universal feature and if it is always observed at the same apparent magnitude, in which case one would suspect that it is the result of background contamination, or at the same absolute magnitude, in which case one would suspect that it is a physical effect. We find from the background contamination test (Section 3.5) that contaminating systems tend to be fainter than $M_{V} \approx-13 \mathrm{mag}$, and so the hump in the case of the NGC 3585 group could at least partially be attributed to contamination.

We fit the observed cumulative LF of each group, for $M_{V}<-10 \mathrm{mag}$, to a cumulative Schechter function (Schechter 1976) using the technique described in Chiboucas et al. (2009). The best-fit faint-end slope, $\alpha=-1.39 \pm 0.03$, for the cumulative LF of the NGC 3585 group is similar to those of other groups (Figure 9(a)). The results for the cumulative LFs of the other groups except for the M96 group with 
$\alpha=-1.36 \pm 0.03$ are introduced in detail in Section 4.2 by Park et al. (2017), and the M101 group is excluded from further comparison because the group shows an abnormal LF shape: very flat for $M_{V}<-10 \mathrm{mag}$ and very steep for $M_{V}>$ $-10 \mathrm{mag}$ (see Bennet et al. 2019 for more detail). We find that the groups have a range of slopes, with the LF slope of the LG $(\alpha \approx-1.0$; Chiboucas et al. 2009; McConnachie et al. 2009; Kim et al. 2011; Ferrarese et al. 2016; Park et al. 2017) being considerably flatter than any of these. On the other hand, all observed faint-end slopes are much flatter than that of the subhalo mass function in the $\Lambda \mathrm{CDM}$ model $(\alpha=-1.8)$ (Trentham \& Tully 2002).

To search for a dependence of the faint-end slope on group properties, we initially investigated a correlation between group mass and LF slope in our previous study (Park et al. 2017). There we suggested that the LF slope flattens as group mass increases. However, the small sample size, the large uncertainties, and the membership problem render this a preliminary result only. Including the new results for the NGC 3585 group and the M96 group in Figure 9 seems to support the previous claim, although we still need more data. To construct this figure, we adopted $\sigma_{\text {group }}$ as an indicator for group mass. The values of $\sigma_{\text {group }}$ for groups other than NGC 3585 and M96 are from Table 4 in Park et al. (2017). The values for the NGC 3585 group, $\sigma_{\text {group }}=70 \mathrm{~km} \mathrm{~s}^{-1}$, and the M96 group, $\sigma_{\text {group }}=$ $233 \mathrm{~km} \mathrm{~s}^{-1}$ listed as M105, are from Makarov \& Karachentsev (2011). The relation between $\alpha$ and $\sigma_{\text {group }}$ can be expressed as $\alpha=0.0003 \sigma_{\text {group }}-1.353$, but a correlation analysis suggests that the confidence with which we can claim a correlation is only at about $1 \sigma$. This marginal nature of the result could be the result of systematic differences among group results obtained from diverse surveys and of small sample statistics as well as of the group membership. A large and uniform survey is required to establish this result. The homogeneous samples obtained from the KMTNet survey of dwarf galaxies in $\gtrsim 30$ groups will serve this purpose.

We close this section by noting the apparent discrepancy between our results and those of Zabludoff \& Mulchaey (2000) and Balogh et al. (2001), who find steeper faint-end slopes in denser, more massive environments. However, these previous results characterize significantly more luminous galaxies than those characterized here (e.g., $-17.8<M_{R}<-19.8$, for Zabludoff \& Mulchaey 2000). If the faint-end LF is not a simple power law, as suggested earlier in this section, then constraining $\alpha$ over different absolute magnitude ranges will lead to apparently conflicting results. Interpreting differences among results from various studies is also complicated by the comparison of LFs derived from spectroscopically confirmed samples, such as that used by Zabludoff \& Mulchaey (2000), and those derived from samples that either apply statistical background corrections or argue that they are minor. It is therefore premature to place much weight on the trend seen in Figure 9 until the larger group survey securely establishes the shape of the faint-end LF and the role of background contamination.

\section{Conclusion and Summary}

We identify a total of 46 new dwarf galaxy candidates in the NGC 3585 group over $7 \square^{\circ}$ using wide and deep images obtained from the KMTNet Supernova Program. We present $B V I$ surface photometry for 50 dwarf galaxy candidates, including 4 previously known dwarf galaxies. For various reasons, including the radial distributions of bright and faint, nucleated and non-nucleated, standard and UDGs, we suggest that the NGC 3585 group may be a dynamically younger system than the typical group. We summarize our results as follows.

1. There is a significant population of dwarf galaxies in the NGC 3585 group. The projected number density of dwarf galaxy candidates decreases exponentially (or with power index, $\alpha \approx-1.5)$ with distance from the center of NGC 3585 and flattens beyond $1.5(\sim 0.5 \mathrm{Mpc})$. The background contamination estimated from this density profile is about $23 \%$, which implies that about 36 of the 46 dwarf galaxy candidates within 1.5 are members of the NGC 3585 group. This background level is similar to the value based on the control field.

2. The internal structure of the NGC 3585 dwarf galaxy candidates is not unusual. Their color and Sérsic- $n$ parameter distributions are consistent with those of dwarfs in other galaxy groups (M81, M83, and NGC 2784). Their color-magnitude relation is also similar to the early-type galaxies in galaxy clusters.

3. There is an unusual lack of radial dependence in the properties of the NGC 3585 dwarf galaxy candidates. The color and the number density profiles do not show a radial dependency. We interpret this result to mean that the NGC 3585 group might be dynamically younger than at least the NGC 2784 group in our previous study.

4. The incidence of nucleated dwarfs may offer an insight into the role of environment in galaxy evolution. We find eight nucleated dwarf galaxy candidates in the NGC 3585 group. The incidence of nucleation appears to be higher at brighter magnitudes and redder colors. We find no radial dependence on the incidence. The incidence of the nucleated dwarf galaxy candidates in this group is roughly $20 \%$, which is much lower than that in galaxy clusters $(60 \%-80 \%)$, and is roughly consistent with what is found in other groups.

5. The radial distribution of UDGs may also be a promising clue. The four UDG candidates we identify in the NGC 3585 group are all within the central region of the group, in contrast with what is found in clusters (van Dokkum et al. 2015; van der Burg et al. 2016). If this group is dynamically young, the UDGs may not yet have suffered sufficiently from tidal disruption.

6. The faint-end slope, $\alpha$, of the galaxy LF correlates weakly with group mass. For NGC 3585 we measure $\alpha \approx-1.39 \pm 0.03$ from the cumulative LF. The faintend slopes of the LFs seem to become flatter as the group masses increase. However, even when the group membership problem is excluded, this correlation is still not highly statistically significant, and systematic uncertainties in the shape of the LF remain. A large homogeneous survey for nearby groups, such as our KMTNet dwarf search project, should establish or refute the existence of this correlation.

The authors are grateful to the staff of the KMTNet. This research has made use of the KMTNet system operated by the Korea Astronomy and Space Science Institute (KASI), and the data were obtained at three host sites of CTIO in Chile, SAAO in South Africa, and SSO in Australia. H.S.P. was supported in part by the National Research Foundation of Korea (NRF) 
grant funded by the Korea government (MSIT, Ministry of Science and ICT; No. NRF-2019R1F1A1058228). D.-S.M. was supported in part by a Leading Edge Fund from the Canadian Foundation for Innovation (project No. 30951) and a Discovery grant from the Natural Sciences and Engineering Research Council of Canada.

\section{ORCID iDs}

Hong Soo Park (1) https://orcid.org/0000-0002-3505-3036 Dae-Sik Moon (ib https://orcid.org/0000-0003-4200-5064 Dennis Zaritsky (i) https://orcid.org/0000-0002-5177-727X Sang Chul Kim (i) https://orcid.org/0000-0001-9670-1546 Youngdae Lee (iD https://orcid.org/0000-0002-6261-1531

\section{References}

Abraham, R. G., \& van Dokkum, P. G. 2014, PASP, 126, 55 Afsariardchi, N., Moon, D.-S., Drout, M. R., et al. 2019, ApJ, 881, 22 Aihara, H., Arimoto, N., Armstrong, R., et al. 2018, PASJ, 70, S4 Antoniadis, J., Moon, D.-S., Ni, Y. Q., et al. 2017, ApJ, 844, 160 APASS 2015, The AAVSO Photometric All-Sky Survey: Data Release 9, https://www.aavso.org/apass/

Balogh, M. L., Christlein, D., Zabludoff, A. I., \& Zaritsky, D. 2001, ApJ, 557,117

Bechtol, K., Drlica-Wagner, A., Balbinot, E., et al. 2015, ApJ, 807, 50

Behroozi, P. S., Conroy, C., \& Wechsler, R. H. 2010, ApJ, 717, 379

Bennet, P., Sand, D. J., Crnojević, D., et al. 2017, ApJ, 850, 109

Bennet, P., Sand, D. J., Crnojević, D., et al. 2019, arXiv:1906.03230

Benson, A. J., Lacey, C. G., Baugh, C. M., Cole, S., \& Frenk, C. S. 2002 MNRAS, 333, 156

Bertin, E. 2006, in ASP Conf. Ser. 351, Automatic Astrometric and Photometric Calibration with SCAMP, ed. C. Gabriel et al. (San Francisco, CA: ASP), 112

Bertin, E., Mellier, Y., Radovich, M., et al. 2002, in ASP Conf. Ser. 281, The TERAPIX Pipeline, ed. D. A. Bohlender, D. Durand, \& T. H. Handley (San Francisco, CA: ASP), 228

Brown, S., Moon, D.-S., Ni, Y. Q., et al. 2018, ApJ, 860, 21

Carlsten, S. G., Beaton, R. L., Greco, J. P., et al. 2019a, ApJL, 878, L16

Carlsten, S. G., Beaton, R. L., Greco, J. P., et al. 2019b, ApJ, 879, 13

Chiboucas, K., Karachentsev, I. D., \& Tully, R. B. 2009, AJ, 137, 3009

Cohen, Y., van Dokkum, P., Danieli, S., et al. 2018, ApJ, 868, 96

Cooper, A. P., Cole, S., Frenk, C. S., et al. 2010, MNRAS, 406, 744

Cortese, L., Bendo, G. J., Isaak, K. G., et al. 2010, MNRAS, 403, L26

Côté, P., Piatek, S., Ferrarese, L., et al. 2006, ApJS, 165, 57

Crnojević, D., Sand, D. J., Spekkens, K., et al. 2016, ApJ, 823, 19

Danieli, S., van Dokkum, P., \& Conroy, C. 2018, ApJ, 856, 69

Danieli, S., van Dokkum, P., Merritt, A., et al. 2017, ApJ, 837, 136

Drlica-Wagner, A., Bechtol, K., Rykoff, E. S., et al. 2015, ApJ, 813, 109

Ferguson, H. C., \& Sandage, A. 1988, AJ, 96, 1520

Ferrarese, L., Côté, P., Sánchez-Janssen, R., et al. 2016, ApJ, 824, 10

Geha, M., Wechsler, R. H., Mao, Y.-Y., et al. 2017, ApJ, 847, 4

Georgiev, I. Y., \& Böker, T. 2014, MNRAS, 441, 3570

Greco, J. P., Greene, J. E., Strauss, M. A., et al. 2018, ApJ, 857, 104

He, M. Y., Moon, D.-S., Neilson, H., et al. 2016, JKAS, 49, 209

Janz, J., Laurikainen, E., Laine, J., Salo, H., \& Lisker, T. 2016, MNRAS, 461, L82
Karachentsev, I. D., Makarov, D. I., \& Kaisina, E. I. 2013, AJ, 145, 101

Kim, E., Kim, M., Hwang, N., et al. 2011, MNRAS, 412, 1881

Kim, S.-L., Lee, C-U., Park, B.-G., et al. 2016, JKAS, 49, 37

Klypin, A., Kravtsov, A. V., Valenzuela, O., \& Prada, F. 1999, ApJ, 522, 82

Lee, M. G., Freedman, W. L., \& Madore, B. F. 1993, ApJ, 417, 553

Lee, M. G., Kang, J., Lee, J. H., \& Jang, I. S. 2017, ApJ, 844, 157

Lee, Y., Moon, D.-S., Kim, S. C., et al. 2019, ApJ, 880, 109

Lee, Y., Park, H. S., Kim, S. C., et al. 2018, ApJ, 859, 5

Lisker, T., Grebel, E. K., \& Binggeli, B. 2008, AJ, 135, 380

Lisker, T., Grebel, E. K., Binggeli, B., \& Glatt, K. 2007, ApJ, 660, 1186

Ludwig, J., Pasquali, A., Grebel, E. K., et al. 2012, AJ, 144, 190

Lupton, R. 2005, Transformations between SDSS magnitudes and other systems, https://www.sdss3.org/dr10/algorithms/sdssUBVRITransform.php/

Makarov, D., \& Karachentsev, I. 2011, MNRAS, 412, 2498

Markwardt, C. B. 2009, in ASP Conf. Ser. 411, Astronomical Data Analysis Software and Systems XVIII, ed. D. A. Bohlender, D. Durand, \& P. Dowler (San Francisco, CA: ASP), 251

McConnachie, A. W. 2012, AJ, 144, 4

McConnachie, A. W., Irwin, M. J., Ibata, R. A., et al. 2009, Natur, 461, 66 Merritt, A., van Dokkum, P., \& Abraham, R. 2014, ApJL, 787, L37

Merritt, A., van Dokkum, P., Danieli, S., et al. 2016, ApJ, 833, 168

Mieske, S., Hilker, M., \& Infante, L. 2003, A\&A, 403, 43

Mihos, J. C. 2016, in IAU Symp. 317, The General Assembly of Galaxy Halos: Structure, Origin and Evolution, ed. A. Bragaglia (Cambridge: Cambridge Univ. Press), 27

Mihos, J. C., Harding, P., Feldmeier, J. J., et al. 2017, ApJ, 834, 16

Miville-Deschênes, M.-A., Duc, P.-A., Marleau, F., et al. 2016, A\&A, 593, A4

Moon, D.-S., Kim, S. C., Lee, J.-J., et al. 2016, Proc. SPIE, 9906, 99064I

Moore, B., Ghigna, S., Governato, F., et al. 1999, ApJL, 524, L19

Müller, O., Jerjen, H., \& Binggeli, B. 2015, A\&A, 583, A79

Müller, O., Jerjen, H., \& Binggeli, B. 2017a, A\&A, 597, A7

Müller, O., Jerjen, H., \& Binggeli, B. 2018, A\&A, 615, A105

Müller, O., Scalera, R., Binggeli, B., \& Jerjen, H. 2017b, A\&A, 602, A119

Pak, M., Rey, S.-C., Lisker, T., et al. 2014, MNRAS, 445, 630

Park, H. S., Moon, D.-S., Zaritsky, D., et al. 2017, ApJ, 848, 19

Presotto, V., Iovino, A., Scodeggio, M., et al. 2012, A\&A, 539, A55

Press, W. H., \& Teukolsky, S. A. 1998, Computers in Physics 2 (Melville, NY: AIP), 74

Rakos, K., \& Schombert, J. 2004, AJ, 127, 1502

Roberts, I. D., Parker, L. C., Joshi, G. D., \& Evans, F. A. 2015, MNRAS, 448, L1

Román, J., \& Trujillo, I. 2017a, MNRAS, 468, 703

Román, J., \& Trujillo, I. 2017b, MNRAS, 468, 4039

Schechter, P. 1976, ApJ, 203, 297

Schlafly, E. F., \& Finkbeiner, D. P. 2011, ApJ, 737, 103

Trentham, N., \& Tully, R. B. 2002, MNRAS, 335, 712

Trentham, N., \& Tully, R. B. 2009, MNRAS, 398, 722

Tully, R. B., Courtois, H. M., Dolphin, A. E., et al. 2013, AJ, 146, 86

Tully, R. B., Libeskind, N. I., Karachentsev, I. D., et al. 2015, ApJL, 802, L25

Turner, M. L., Côté, P., Ferrarese, L., et al. 2012, ApJS, 203, 5 van der Burg, R. F. J., Muzzin, A., \& Hoekstra, H. 2016, A\&A, 590, A20

van Dokkum, P. G., Abraham, R., Merritt, A., et al. 2015, ApJL, 798, L45

Watkins, A. E., Mihos, J. C., Harding, P., et al. 2014, ApJ, 791, 38

Watkins, A. E., Mihos, J. C., \& Harding, P. 2015, ApJL, 800, L3

Wetzel, A. R., Tinker, J. L., \& Conroy, C. 2012, MNRAS, 424, 232

Young, T., Jerjen, H., López-Sánchez, Á. R., \& Koribalski, B. S. 2014, MNRAS, 444, 3052

Zabludoff, A. I., \& Mulchaey, J. S. 2000, ApJ, 539, 136

Zaritsky, D., Donnerstein, R., Dey, A., et al. 2019, ApJS, 240, 1 